\title{
Article
}

\section{The Expression Characteristics of NPF Genes and Their Response to Vernalization and Nitrogen Deficiency in Rapeseed}

\author{
Hongbo Chao ${ }^{1}$, Jianjie $\mathrm{He}^{2}$, Qianqian Cai ${ }^{1}$, Weiguo Zhao ${ }^{2,3}$, Hong Fu ${ }^{1}$, Yingpeng Hua ${ }^{1}$, Maoteng $\mathrm{Li}^{2, *}$ \\ and Jinyong Huang ${ }^{1, *}$ \\ 1 School of Agricultural Sciences, Zhengzhou University, Zhengzhou 450001, China; \\ chaohongbo@zzu.edu.cn (H.C.); caiqianqianhn@163.com (Q.C.); 15290885289@163.com (H.F.); \\ yingpenghua@zzu.edu.cn (Y.H.) \\ 2 Department of Biotechnology, College of Life Science and Technology, \\ Huazhong University of Science and Technology, Wuhan 430074, China; hejianjie@hust.edu.cn (J.H.); \\ zhaoweiguo0517@126.com (W.Z.) \\ 3 Hybrid Rape Research Center of Shaanxi Province, Yangling 712100, China \\ * Correspondence: limaoteng426@hust.edu.cn (M.L.); jinyhuang@zzu.edu.cn (J.H.)
}

Citation: Chao, H.; He, J.; Cai, Q.; Zhao, W.; Fu, H.; Hua, Y.; Li, M.;

Huang, J. The Expression

Characteristics of NPF Genes and Their Response to Vernalization and Nitrogen Deficiency in Rapeseed. Int. J. Mol. Sci. 2021, 22, 4944. https:// doi.org/10.3390/ijms22094944

Academic Editor: Scott D. Russell

Received: 15 April 2021

Accepted: 3 May 2021

Published: 6 May 2021

Publisher's Note: MDPI stays neutral with regard to jurisdictional claims in published maps and institutional affiliations.

Copyright: (c) 2021 by the authors. Licensee MDPI, Basel, Switzerland. This article is an open access article distributed under the terms and conditions of the Creative Commons Attribution (CC BY) license (https:/ / creativecommons.org/licenses/by/ $4.0 /)$.

\begin{abstract}
The NITRATE TRANSPORTER 1/PEPTIDE TRANSPORTER FAMILY (NPF) genes, initially characterized as nitrate or peptide transporters in plants, are involved in the transport of a large variety of substrates, including amino acids, nitrate, auxin (IAA), jasmonates (JAs), abscisic acid (ABA) and gibberellins (GAs) and glucosinolates. A total of 169 potential functional NPF genes were excavated in Brassica napus, and they showed diversified expression patterns in 90 different organs or tissues based on transcriptome profile data. The complex time-serial expression changes were found for most functional NPF genes in the development process of leaves, silique walls and seeds, which indicated that the expression of Brassica napus NPF (BnaNPF) genes may respond to altered phytohormone and secondary metabolite content through combining with promoter element enrichment analysis. Furthermore, many BnaNPF genes were detected to respond to vernalization with two different patterns, and 20 BnaNPF genes responded to nitrate deficiency. These results will provide useful information for further investigation of the biological function of BnaNPF genes for growth and development in rapeseed.
\end{abstract}

Keywords: Brassica napus; NPF gene; transfer protein; gene expression; growth and development

\section{Introduction}

Plant NPF (NITRATE TRANSPORTER 1/PEPTIDE TRANSPORTER FAMILY) proteins display sequence homology with the proton-coupled oligopeptide transporter (POT) family of peptide transporters, belong to the large peptide transporter (PTR) family [1] and are involved in dietary nitrogen absorption in the form of di- and tripeptides [2,3]. In plants, NPF members are initially characterized as nitrate or peptide transporters. AtNPF6.3, known as AtNRT1.1, is the first plant member discovered as a nitrate transporter in Arabidopsis [4], and the crystal structure of AtNPF6.3 recently determined showed the similarity with PTRs in the bacterial $[5,6]$. Subsequently, some NPF members are identified in different plants and demonstrated that they behave as dipeptide transporters [7,8]. So, nitrate/peptide transport function is believed to be a specific feature of this family in plants over a period of time. However, in recent years, several studies demonstrated that some NPF members could transport an even wider range of substrates, including nitrite, chloride, auxin (IAA), abscisic acid (ABA), jasmonates (JAs), and gibberellins (GAs) and glucosinolates [9-12]. Additionally, some of the NPF members are even able to transport more than one different substrate: nitrate/IAA, nitrate/ABA, nitrate/glucosinolates, peptides/amino acids, or GA/JA.

After identifying the first nitrate transporter NPF6.3/NRT1.1, Tsay et al. [4,8] subsequently characterized more than half of the NPF nitrate transporters. So far, more than 
21 NPF members have been demonstrated to be able to transport nitrate in Arabidopsis, many of which are able to transport other substrates, such as ABA, GA, JA and glucosinolates $[8,13]$. Screening 45 out of 53 AtNPF members using a modified yeast two-hybrid system with ABA, GA and JA-Ile specific receptor complexes, Chiba et al. [14] confirmed that nine NPF members had an ABA transport function, 18 NPF members were able to transport GA and 13 NPF members were able to transport the bioactive JA/JA-Ile. NRT1.1/NPF6.3 was confirmed to be able to transport IAA and 2,4-D [15,16]. In addition, PTR3/NPF5.2, PTR1/NPF8.1, PTR5/NPF8.2 and PTR2/NTR1/NPF8.3 were identified as dipeptide and tripeptide transporters using complementation of yeast strains deficient for peptide uptake $[17,18]$.

Based on the phylogenetic relationship, NPF members were divided into eight subfamilies (from NPF1 to NPF8) [1]. In the NPF1 subfamily, MtNPF1.7, from Medicago truncatula, has been characterized to be involved in nodulation and root architecture and behaves as a high-affinity nitrate transporter [19-21]. NPF1.1 and NPF1.2, from Arabidopsis, are confirmed to be important for redistributing xylem-borne nitrate to enhance plant growth [22]. The NPF2 subfamily contains nitrate, phytohormone and glucosinolates transporters, and NPF2 members show a wide range of tissue and developmental specificity in Arabidopsis. AtNPF2.3, as a root stele transporter, contributes to nitrate translocation to shoots under salt stress [23], and the low-affinity nitrate transporter AtNPF2.12 is responsible for nitrate-dependent early embryo development [24]. Besides, five AtNPF members that are capable of transporting glucosinolates belong to the NPF2 subfamily [11]. AtNPF3.1 is the only member of the NPF3 subfamily in Arabidopsis and plays the role of nitrite transport [25]. It has been demonstrated that ABA could be transported by NPF4 subfamily members [10], and some members of the NPF4 subfamily are able to transport GA, such as NPF4.1 and NPF4.2. NPF5 is the largest NPF subfamily and contains numerous members involved in nitrate, ABA, GA, JA and di-peptides transport [14,26,27]. In the NPF6 subfamily, most of the members have been demonstrated to be nitrate transporters, including the first identified NPF member AtNPF6.3 [4,28-30]. At least two different substrates were able to be transported by NPF7 subfamily members based on three well functionally characterized proteins: AtNPF7.2/NTR1.8 and AtNPF7.3/NTR1.5 for nitrate transport [31,32], as well as OsNPF7.3 for dipeptide transport [33]. Three Arabidopsis NPF8 subfamily members, AtNPF8.1/PTR1, AtNPF8.2/PTR5 and AtNPF8.3/PTR2, have been proved to be dipeptide transporters, while AtNPF8.1 and AtNPF8.2 are able to transport JA-Ile simultaneously [34,35]. It is noteworthy that 38 and 17 NPF genes have been characterized at the functional level in Arabidopsis and rice, respectively, and more than half of them function on nitrate transport and are distributed on eight NPF subfamilies, and play important roles in nitrate absorption, translocation and utilization [13,31,36,37]. Generally, $N P F$ transporters with a very broad substrate specificity have an important function in plant growth and development, and genome-wide identification has been implemented in poplar [38], alfalfa [36], apple [39] and wheat [13], but a few systematic analyses have been conducted for NPF genes in Brassica species.

B. napus, an allotetraploid evolved from the hybridization between two diploid progenitors (Brassica rapa and Brassica oleracea), is an important oil crop in the world. Compared with Arabidopsis, B. napus has experienced a whole-genome triplication, which occurred between 7.9 and 14.6 million years ago [40], and a hybridization event via the natural crossing of B. rapa and B. oleracea (both of which diverged from a common ancestor $\sim \mathrm{n} 4$ million years ago) about 7500 years ago [41-43]. Recently, the genomes of 'Darmor- $b z h$ ' and 'ZS11' have been successfully sequenced and assembled [42,44], and Pan-genomes have been constructed based on next-generation sequencing technologies for B. napus, which facilitate systematically excavating NPF genes in rapeseed. Although the NPF genes have been identified in rapeseed [45,46], functional NPF proteins and genes should be excavated, and their expression characteristics and role in the tissues that play an important role in rapeseed oil yield should be further analyzed. In this study, 169 NPF genes were excavated in the B. napus, and the expression profiles of BnaNPF genes in diverse tissues, as well as 
time-serial expression changes in leaf, silique wall and seed, were determined; meanwhile, expression changes induced by vernalization at different development stages and response to nitrate deficiency were analyzed. These results will provide useful information for further investigation of the biological function of BnaNPF genes for growth and development in B. napus.

\section{Results}

\subsection{Distribution and Synteny Analysis of NPF Genes in Four Brassica Species}

Based on BLASTP using 53 Arabidopsis NPF protein and phylogenetic analysis (Figure S1), a total of 169 NPF genes encoding 186 proteins were identified in the B. napus genome. To investigate the evolution of BnaNPF genes, the synteny of NPF gene pairs between the B. rapa and Arabidopsis genome, B. oleracea and Arabidopsis genome, B. napus and B. rapa genome, and the B. napus and B. oleracea was performed to further understand the expansion mechanism of NPF genes in B. napus (Figure 1). The result shows that most of the $B n a N P F$ genes exhibited evolutionary and syntenic relationships with NPF genes in Arabidopsis, B. rapa, and B. olereaca (Figure S2), suggesting the contribution to the evolution of the $B n a N P F$ gene family. Furthermore, the Ka (nonsynonymous nucleotide substitution rate), Ks (synonymous nucleotide substitution rate) and $\mathrm{Ka} / \mathrm{Ks}(\mathrm{Ka} / \mathrm{Ks}$ ratio) of orthologous pairs on BnaNPF and AtNPF genes were calculated to test the evolutionary selection pressure (Table S2). The majority of orthologous BnaNPF gene pairs had $\mathrm{Ka} / \mathrm{Ks}<1$, which suggested that most of the BnaNPF genes have undergone purifying selection to preserve gene function. The mean value of NPF3 $(\mathrm{Ka} / \mathrm{Ks}=0.10), N P F 6(\mathrm{Ka} / \mathrm{Ks}=0.11)$ and NPF7 $(\mathrm{Ka} / \mathrm{Ks}=0.13)$ gene pairs was lower than other subfamilies, showing that these three subfamilies may have suffered robust purifying selective pressure during evolution. However, some of the BnaNPF genes had $\mathrm{Ka} / \mathrm{Ks}>1$, including BnaA01NPF2.8, BnaC01NPF2.9, BnaA06NPF2.10, BnaC03NPF2.12 and BnaC01NPF2.25 in the NPF2 subfamily, BnaA09NPF4.15 in the NPF4 subfamily, BnaA05NPF5.1, BnaC04NPF5.3, BnaC03NPF5.7, BnaA03NPF5.8, BnaA02NPF5.15, BnaA02NPF5.40, BnaC02NPF5.41 and BnaC06NPF5.42 in the NPF5 subfamily, and BnaC09NPF 8.19 in the NPF8 subfamily, suggesting that these BnaNPF genes are subjected to positive selection during the evolution from Arabidopsis to rapeseed.

The distribution and synteny of NPF genes were marked on the chromosomes of B. rapa, B. oleracea and B. napus (Figure 1b). NPF genes are unevenly distributed on every chromosome, and often organized as clusters in the genome of $B$. rapa, B. oleracea and B. napus. In the B. napus genome, the chromosomes A09 and C06 possess the most BnaNPF genes (15, respectively), and A08 possess only four BnaNPF genes, which were clustered on the chromosome terminal. NPF genes distributed on the B. rapa and B. oleracea genome keep good collinearity with NPF genes on the A and C sub-genome of B. napus, respectively. B. rapa genome contains 82 NPF genes, and the corresponding A sub-genome of $B$. napus contains only 76 NPF genes; the B. oleracea genome contains 70 NPF genes, and the corresponding $C$ sub-genome of $B$. napus contains 93 NPF genes, which indicates that parts of NPF genes from $B$. rapa genome were lost or recombined to the $C$ genome of $B$. napus in the evolution process. For example, BraNPF5.21 on the terminal of the chromosome BraA05 was replicated and recombined to BnaC05 chromosome (BnaC05NPF5.37 and $B n a C 05 N P F 5.38$ ). According to the synteny analysis, 97 BnaNPF genes evolved from the $B$. rapa genome, and $72 \mathrm{BnaNPF}$ genes from the B. oleracea genome. Furthermore, $73 \mathrm{BraNPF}$ genes retained synteny with NPF genes in the B. napus genome, including $55 \mathrm{BraNPF}$ genes with a 1:1 synteny relationship, 16 BraNPF genes with a 1:2 relationship (duplication in B. napus genome) and even two BraNPF genes with more than a 1:2 relationship (1:3 and 1:5) (Tables 1 and S3). Nine BraNPF orthologs were not identified in the B. napus genome (1:0 relationship) and two BnaNPF orthologs were not identified in the B. rapa genome (0:1 relationship), suggesting a loss of the gene during evolution. Sixty-one BolNPF genes retained synteny with NPF genes in the B. napus genome, including 54 with a 1:1 relationship and 7 with a 1:2 relationship. Twenty-six and five translocations were identified for NPF genes when comparing the B. napus genome with the B. rapa and $B$. 
oleracea genome, respectively. Besides, because the genomic data of $B$. napus have not yet been fully mapped to the chromosome, the chromosomal location and evolution of three BnaNPF genes (BnaNPF2.26, BnaNPF2.29 and BnaNPF2.30) is still unclear.

Table 1. The synteny relationship of NPF genes between B. rapa and B. napus, and between B. oleracea and B. napus.

\begin{tabular}{ccccccc}
\hline Ratio $^{\text {a }}$ & $\mathbf{0 : 1}$ & $\mathbf{1 : 0}$ & $\mathbf{1 : 1}$ & $\mathbf{1 : 2}$ & $\mathbf{1 : 3}$ & $\mathbf{1 : 5}$ \\
\hline B. rapa & 2 & 9 & 55 & 16 & 1 & 1 \\
B. oleracea & 4 & 9 & 54 & 7 & &
\end{tabular}

${ }^{a}$ Orthologous NPF gene ratio by comparing $B$. rapa and B. oleracea with the B. napus genome. 0:1 represents NPF orthologs lost in the B. rapa or B. oleracea genome, 1:0 represents NPF orthologs lost in the B. napus genome, and $1: 2,1: 3$ and 1:5 represent different replication multiples in the B. napus genome.

\subsection{B. napus Genome Possessed the Most NPF Genes}

Using the sequences of 53 Arabidopsis NPF family protein as queries to perform BLASTp and the information from the article Leran et al. [1] reported, NPF proteins from 36 sequenced species were retrieved, including B. rapa, B. oleracea and B. napus. Information regarding genome size and number of NPF genes is shown in Table 2. The genome sizes of these 34 plant species ranged from $127.42 \mathrm{Mb}$ (Arabidopsis) to $2271.03 \mathrm{Mb}$ (Zea mays), and the NPF gene number varied from 23 (Physcomitrella patens) to 169 (B. napus). The $B$. napus genome possessed the most NPF genes (167), though its genome size was smaller than that of Malus domestica and Zea mays, which indicated that the copy number variations of $B$. napus NPF genes might be attributed to their requirement for (un)specific substrates as a result of evolutionary selection, such as some NPF2 members for transporting glucosinolates [8]. All NPF genes were grouped into eight clades with known 53 NPF members from Arabidopsis. Most plants have more NPF2, NPF4 and NPF5 subfamily members. NPF1 and NPF2 subfamilies are absent from the two lower plants Physcomitrella patens and Selaginella moellendorffii. In addition, based on the BnPIR database that provides more detailed annotation for $B$. napus genes, 11 BnaNFP genes were identified to encode two proteins derived from two different transcripts, and three BnaNFP genes encode three proteins translated from three different transcripts. Therefore, a total of 186 BnaNPF proteins were identified in B. napus, including 17 proteins from different transcripts. Based on the phylogenetic tree (Figure S1), the evolutionary relationship of NPF proteins between B. napus and Arabidopsis was easy to compare and provided a good guide for studying the function of NPF genes in B. napus. According to known Arabidopsis NPF protein subfamily information and phylogenetic tree branches, eight unambiguous clades that represented eight $B$. napus NPF subfamilies were identified. The BnaNPF5 subfamily was the largest because of a larger number of Arabidopsis NPF5 members and possessed 63 members (more than a third of the total number of BnaNPF genes), followed by NPF2 (30), NPF8 (19), NPF4 (16), NPF6 (15), NPF7 (10), NPF1 (10), and NPF3 (6). Additionally, BnaA05NPF5.1 and BnaC04NPF5.2, located in the same branch with AtNPF5.1, were grouped into the NPF2 clade, which suggested that the two NPF genes might be more closely related to $B$. napus NPF2 in evolution. Similarly, BnaA02NPF6.14 and BnaC02NPF6.15 seemed to be more closely related to NPF7. Most of the phylogenetic branches within the same clade showed a high bootstrap value $(>0.80)$, which reflected the low genetic differentiation of Arabidopsis and B. napus NPF genes within the subfamily. 

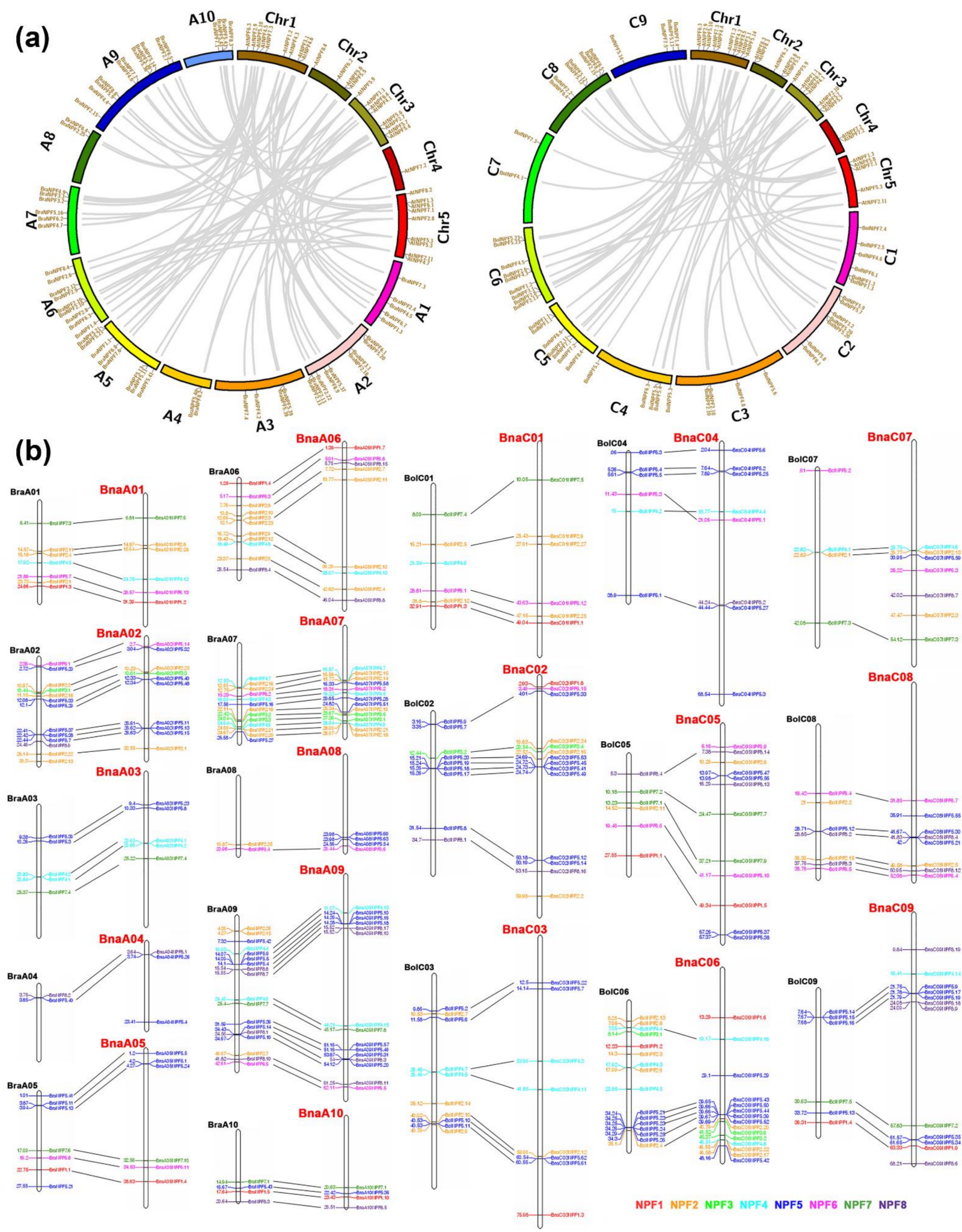

NPF1 NPF2 NPF3 NPF4 NPF5 NPF6 NPF7 NPF8

Figure 1. Genomic distribution of the NPF genes in Arabidopsis, B. rapa, B. oleracea and B. napus, and synteny of the NPF genes in the four Brassica species genomes. (a) The synteny of NPF genes between Arabidopsis and B. rapa (Left), and between Arabidopsis and B. oleracea (Right). (b) The collinearity of NPF genes between B. rapa and the A sub-genome of $B$. napus, and between B. oleracea and the $C$ sub-genome of $B$. napus. Three letters before the chromosome were used to distinguish the species, and the color of the font on the bottom right distinguishes the subfamilies. 
Table 2. Copy number variations (CNVs) of the NPF genes in 36 plant species.

\begin{tabular}{|c|c|c|c|c|c|c|c|c|c|c|}
\hline Organism Name & NPF1 & NPF2 & NPF3 & NPF4 & NPF5 & NPF6 & NPF7 & NPF8 & Total & $\begin{array}{l}\text { Genome } \\
\text { Size }(\mathrm{Mb})\end{array}$ \\
\hline Arabidopsis lyrata (D) & 3 & 14 & 1 & 9 & 17 & 4 & 3 & 5 & 56 & 202.97 \\
\hline Arabidopsis thaliana (D) & 3 & 14 & 1 & 7 & 16 & 4 & 3 & 5 & 53 & 127.42 \\
\hline Aquilaria agallochum (D) & 6 & 7 & 3 & 12 & 13 & 5 & 3 & 6 & 55 & 726.71 \\
\hline Brachypodium distachyon $(\mathrm{M})$ & 2 & 6 & 4 & 13 & 21 & 8 & 11 & 17 & 82 & 271.3 \\
\hline Brassica rapa (D) & 4 & 23 & 3 & 9 & 23 & 7 & 5 & 8 & 82 & 401.93 \\
\hline Brassica oleracea (D) & 4 & 15 & 2 & 8 & 26 & 6 & 5 & 4 & 70 & 554.98 \\
\hline Brassica napus (D) & 10 & 30 & 6 & 16 & 63 & 15 & 10 & 19 & 169 & 976.19 \\
\hline Carica papaya (D) & 4 & 14 & 3 & 8 & 12 & 8 & 6 & 4 & 59 & 370.42 \\
\hline Capsella rubella (D) & 3 & 12 & 1 & 6 & 17 & 4 & 3 & 5 & 51 & 133.06 \\
\hline Citrus clementina (D) & 9 & 7 & 3 & 9 & 17 & 6 & 4 & 4 & 59 & 301.37 \\
\hline Citrus sinensis (D) & 8 & 7 & 3 & 10 & 17 & 6 & 4 & 4 & 59 & 319.23 \\
\hline Cuscuta campestris (D) & 4 & 9 & 3 & 8 & 19 & 6 & 5 & 5 & 59 & 476.79 \\
\hline Eucalyptus grandis (D) & 6 & 12 & 4 & 11 & 19 & 6 & 4 & 6 & 68 & 691.43 \\
\hline Fragaria vesca (D) & 0 & 13 & 2 & 8 & 23 & 3 & 5 & 6 & 60 & 214.37 \\
\hline Glycine $\max (\mathrm{D})$ & 13 & 14 & 6 & 22 & 41 & 11 & 14 & 13 & 134 & 927.71 \\
\hline Gossypium raimondii (D) & 7 & 10 & 4 & 14 & 14 & 11 & 7 & 8 & 75 & 773.77 \\
\hline Linum usitatissimum (D) & 12 & 7 & 4 & 14 & 25 & 9 & 11 & 10 & 92 & 316.17 \\
\hline Malus domestica (D) & 2 & 34 & 4 & 21 & 44 & 17 & 8 & 9 & 139 & 1874.77 \\
\hline Manihot esculenta (D) & 7 & 12 & 6 & 10 & 23 & 7 & 5 & 5 & 75 & 292.1 \\
\hline Medicago truncatula (D) & 8 & 12 & 3 & 14 & 25 & 8 & 9 & 1 & 80 & 412.92 \\
\hline Oryza sativa $(\mathrm{M})$ & 3 & 6 & 5 & 12 & 29 & 6 & 11 & 21 & 93 & 389.75 \\
\hline Phaseolus vulgaris (D) & 8 & 11 & 3 & 12 & 22 & 5 & 7 & 6 & 74 & 521.08 \\
\hline Populus trichocarpa (D) & 15 & 9 & 5 & 12 & 26 & 6 & 5 & 7 & 85 & 434.29 \\
\hline Prunus persica (D) & 2 & 15 & 1 & 8 & 16 & 5 & 5 & 5 & 57 & 214.22 \\
\hline Ricinus communis (D) & 5 & 20 & 3 & 7 & 13 & 5 & 4 & 3 & 60 & 350.62 \\
\hline Setaria italica $(\mathrm{M})$ & 4 & 11 & 8 & 16 & 19 & 7 & 12 & 21 & 98 & 405.87 \\
\hline Solanum tuberosum (D) & 17 & 10 & 2 & 15 & 8 & 9 & 4 & 8 & 73 & 772.25 \\
\hline Solanum lycopersicum (D) & 19 & 16 & 2 & 12 & 11 & 12 & 7 & 11 & 90 & 760.07 \\
\hline Sorghum bicolor $(\mathrm{M})$ & 4 & 8 & 7 & 16 & 22 & 6 & 9 & 19 & 91 & 709.35 \\
\hline Theobroma cacao (D) & 4 & 14 & 3 & 10 & 19 & 7 & 4 & 5 & 66 & 345.99 \\
\hline Vitis vinifera $(\mathrm{D})$ & 4 & 7 & 2 & 6 & 21 & 5 & 4 & 3 & 52 & 486.2 \\
\hline Zea mays $(\mathrm{M})$ & 4 & 4 & 6 & 12 & 17 & 8 & 12 & 16 & 79 & 2271.03 \\
\hline Amborella trichopoda (D) & 1 & 5 & 2 & 7 & 15 & 4 & 3 & 7 & 45 & 706.60 \\
\hline Physcomitrella patens (L) & 0 & 0 & 1 & 1 & 8 & 6 & 3 & 4 & 23 & 472.081 \\
\hline Selaginella moellendorffii (L) & 0 & 0 & 4 & 4 & 11 & 6 & 5 & 16 & 46 & 212.315 \\
\hline Selaginella moellendorffii (L) & 0 & 0 & 4 & 4 & 11 & 6 & 5 & 16 & 46 & 212.315 \\
\hline
\end{tabular}

D dicots, $\mathrm{M}$ monocots, $\mathrm{L}$ lower plants.

\subsection{BnaNPF Gene Owning the PTR2 Functional Domain Might Be Regulated by Multiple Phytohormones}

The gene structures (number and organization exon-intron) are typical evolutionary imprints within certain gene families and are closely related to their function. The exon/intron arrangements of 169 BnaNPF genes were analyzed together with 53 AtNPF by comparing CDS and the corresponding genomic DNA sequences within and between subgroups based on the phylogenetic tree (Figure S3). The BnaNPF genes have a higher degree of divergence among gene structure than NPF genes in Arabidopsis and contained the numbers of exons varying from 2 to 18. BnaC02NPF1.8 and BnaC09NPF1.9 in the NPF1 subfamily and BnaC05NPF2.6, BnaA06NPF2.7 and BnaA06NPF6.8 in the BnaNPF2 subfamily were significantly longer than other genes and contained the most exons (16, $16,18,18$ and 8, respectively); however, most of the BnaNPF genes contained no more than six exons. BnaNPF genes in different branches exhibited different gene structural features, while the genes in the same branch generally had similar intron/exon distribution patterns. For instance, BnaA05NPF1.4, BnaC05NPF1.5 and BnaA06NPF1.7 in the BnaNPF1 subgroup, BnaC05NPF5.56, BnaA09NPF5.57, BnaA07NPF5.58 and BnaC07NPF5.59 in the BnaNPF5 subgroup, and BnaC07NPF7.3, BnaA03NPF7.4, BnaC01NPF7.5 and BnaA01NPF7.6 
in the BnaNPF7 subgroup had almost the same exon/intron distribution characteristics, and different distribution patterns were found between subgroups. To further explore the specific and conserved regions of 186 BnaNPF proteins, four conserved domains, PTR2, MFS_1 (Major facilitator family), Chorismate_bind and PDDEXK_6 (PDDEXK-like family of unknown function), were identified by the HMMER (biosequence analysis using profile hidden Markov models) website (Figure S3). PTR2 domain, responsible for protondependent transport, is the signature domain of NPF protein and could be found in each BnaNPF member, suggesting functional conservation. The major facilitator superfamily MFS_1 domain feature was detected to partially overlap or exist within the PTR2 domain in some BnaNPF members $(45 / 186)$. The chorismite_bind domain involved in chorismateutilizing was found in BnaC05NPF2.6 and BnaA06NPF2.7, and BnaC03NPF4.4 contained an unknown function PDDEXK_6 domain.

Transcription factors bind to CREs in the promoter and regulate the expression of the target genes [47]. Generally, genes with similar CREs show the same expression patterns. The 2.0-kb upstream regulatory regions of the BnaNPF genes were used to explore the CREs (Figure 2 and Table S4). The results show that 157 BnaNPF genes contained at least one type of CRE in the promoter regions, which indicated that complex transcriptional regulation might be implicated for BnaNPF genes. Apart from the common CREs, such as the CAAT-box, TATA-box and some light-responsive elements (G-box, Box 4, GT1-motif and TCT-motif), some phytohormone-responsive elements, such as the auxin-responsive elements (TGA-element, AuxRR-core, GATA-box, TGA-box and AuxRE), the ABA-responsive element (ABRE) and the JA-responsive elements (CGTCA-motif and TGACG-motif), and some abiotic stress-responsive elements, such as the low-temperatureresponsive element (LTR), the salicylic acid-responsive element (TCA-element) and the anaerobic-responsive element (ARE), were identified. Some over-presented CREs, including ARE, ABRE, CGTCA-motif, TGACG-motif, LTR and TC-rich repeats, were involved in the molecular response of plants to phytohormone, defense and stress responsiveness (Figure 2a). Among these, the MYB recognition site was most enriched, implying that the MYB transcript factors may play crucial roles in the transcriptional regulation of the $B n a N P F$ genes. Besides, RY-element, the CRE involved in seed-specific regulation, was identified in the promoters of the 15 BnaNPF genes, which indicated that these BnaNPF genes might function in the process of seed development and matter storage.

\subsection{Gene Expression Pattern Analysis of NPF Genes in Diverse Tissues of B. napus}

In order to explore the potential tissues in which NPF genes function in B. napus, the expression profiles were characterized in 90 different organs or tissues, including cotyledon, root, vegetative rosette, stem peel (peel of upper, middle and lower stem), leaf (23 parts or periods), sepal, petal, filament, pollen, bud, silique wall (30 development periods) and seed (24 development periods) based on transcriptome information from BnTIR (http:/ / yanglab.hzau.edu.cn/BnTIR/eFP, Accessed on 4 May 2021). Except for half of the genes in the BnaNPF2, BnaNPF5 and BnaNPF8 subfamily that has relatively low expression values $(\mathrm{FPKM}<1)$ or no expression, most of the BnaNPF genes had preferential expression profiles in the 90 tissues (Figure 3). For instance, half of the BnaNPF1 genes showed high expression levels in the silique wall at the early and middle development stages and in leaves of all parts; one-third of BnaNPF2 genes (10/30) showed specific expression in the seeds at early and middle development stages; most of the BnaNPF7 genes $(8 / 10)$ were highly expressed in the bud, petal, pollen and seeds. In general, expression patterns were conserved in each clade within a subfamily, but were quite different across different subfamilies, suggesting the expression differentiation trend of this gene family. For instance, expression patterns of BnaNPF2 and BnaNPF4 subfamilies were classified into three conserved patterns that were consistent with the three major clades in these two subfamilies, and while the expression profile of the BnaNPF3 genes was similar in this subfamily. 
a
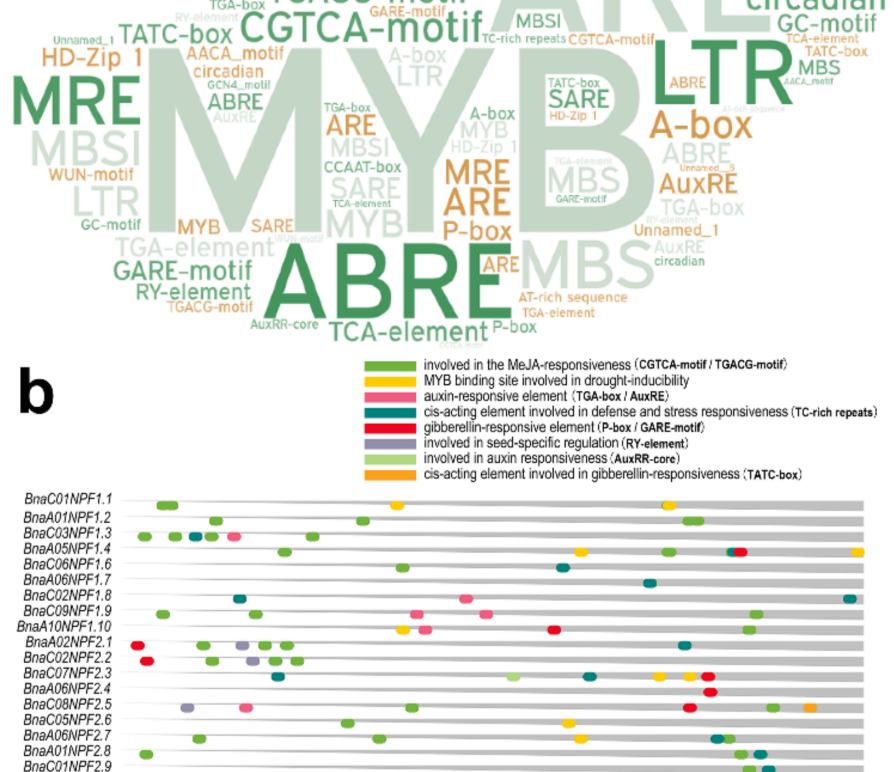

-


Figure 2. Identification of the CREs of the BnaNPF genes. (a) Over-presentation of the CREs in the promoters of the 157 BnaNPF genes. The bigger the font size, the more frequent the CRE appears in BnaNPF genes. (b) Genomic distribution and relative abundance of the 8 kinds of CREs involved in the molecular response of plants to phytohormone, abiotic stress responsiveness and seed-specific regulation in the BnaNPF gene promoters. Different kinds of CREs are indicated with different colors. 
(a)
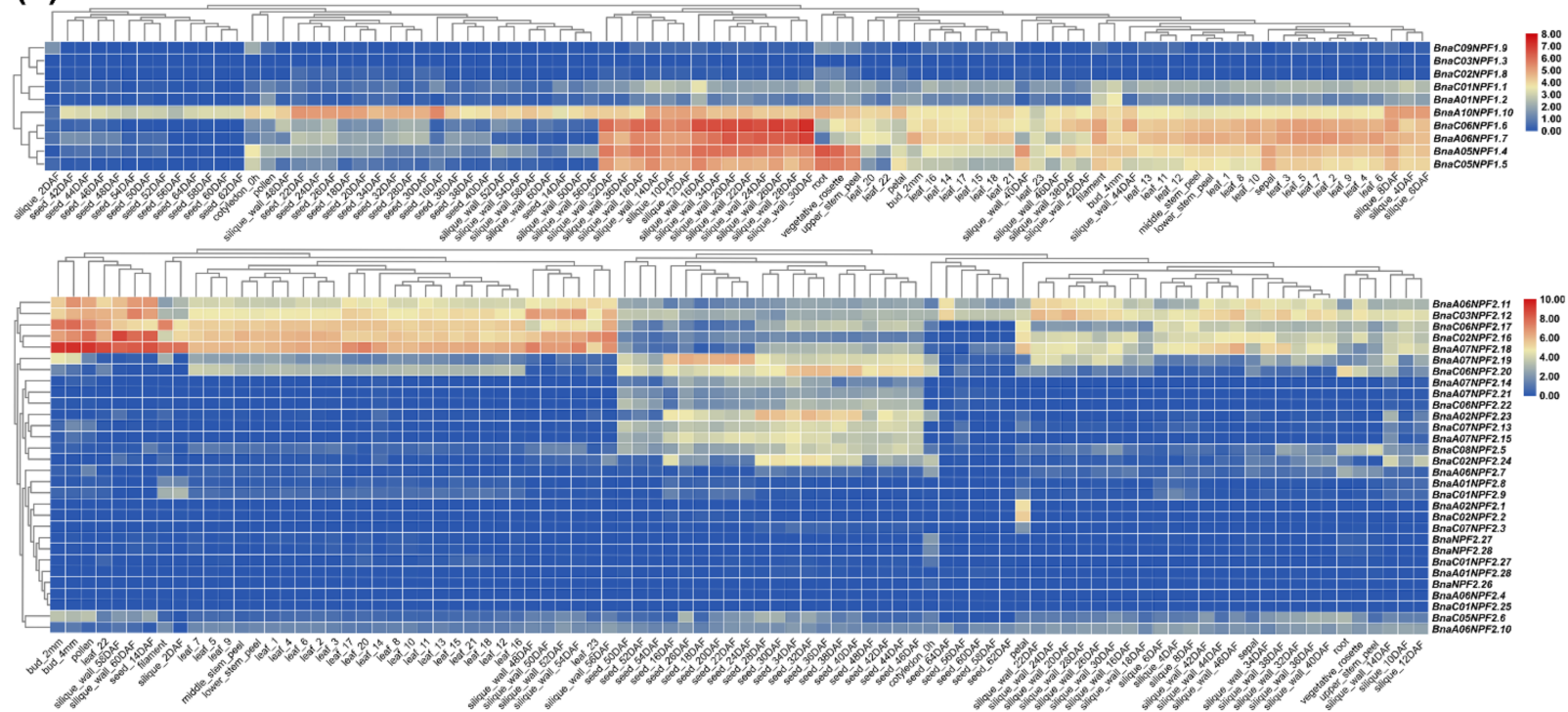

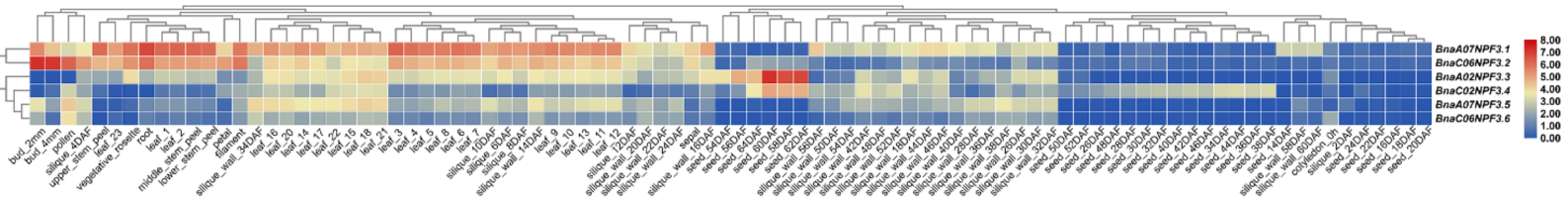

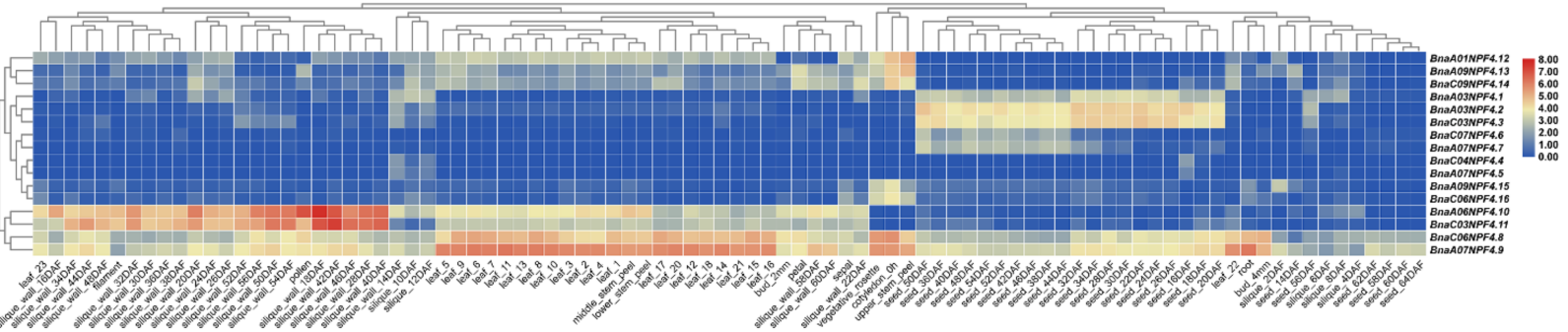

(b)

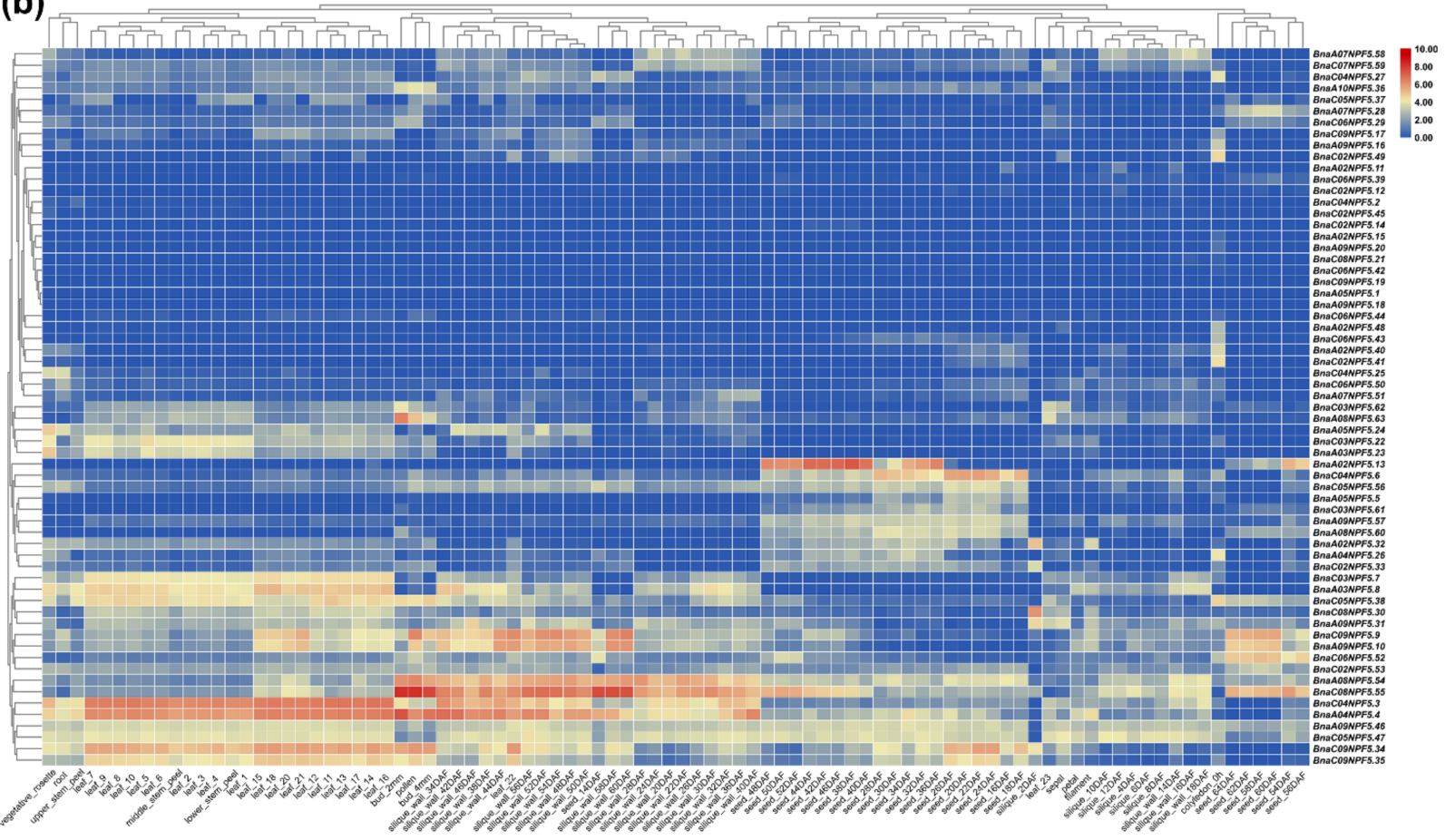

Figure 3. Cont. 


\section{(c)}

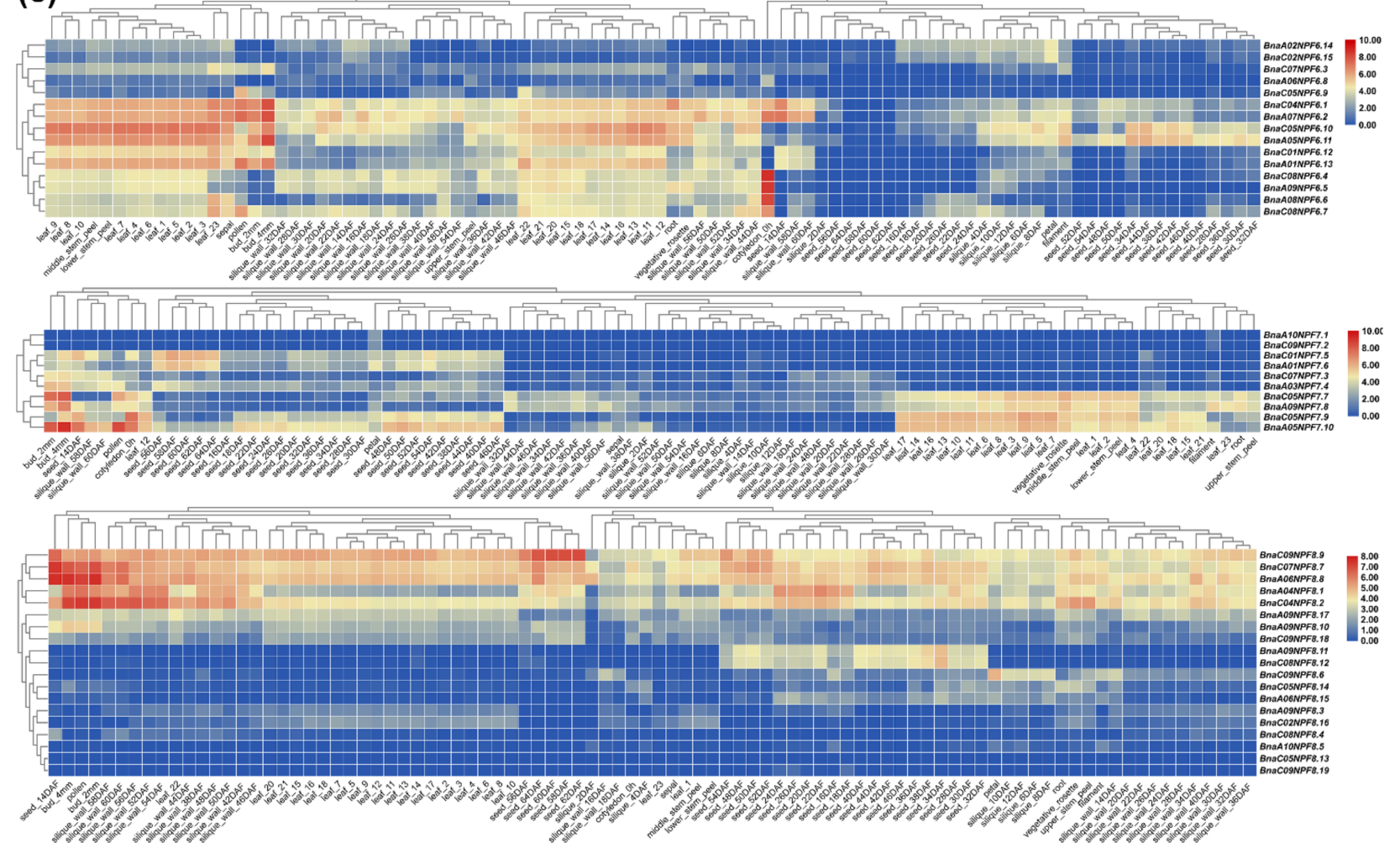

Figure 3. Gene expression profiles of NPF genes for 90 tissues or periods in B. napus. (a-c) display the gene expression profiles of NPF1-4, NPF5, and NPF6-8 genes, respectively. The word "DAF" and the number before it mean the days after flowering. The number following "leaf" means leaf in different growth stages and parts of the plant, which originates from the online website (http:/ / yanglab.hzau.edu.cn/BnTIR/eFP, Accessed on 4 May 2021).

Based on the expression profiles in seeds, silique wall and leaves from multiple development periods or plant parts, the expression patterns of BnaNPF genes in leaves, silique wall and seeds could be clarified clearly (Figure 4). Although some members of both BnaNPF1 (4/10) and BnaNPF2 (5/30) were highly expressed in the silique wall of the developing silique, BnaNPF1 genes showed higher expression levels at the middle development stages, and BnaNPF2 genes were higher expressed at the later development stages. The members of BnaNPF3 with high expression levels in the silique wall, BnaA07NPF3.1 and BnaC06NPF3.2, were higher expressed at the early than later development stage of the silique. However, some members of BnaNPF4 and BnaNPF5, such as BnaA06NPF4.10, BnaC03NPF4.11, BnaC04NPF5.3, BnaA04NPF5.4, BnaA08NPF5.54 and BnaC08NPF5.55, were higher expressed in silique wall at the later than early development stages of the silique. BnaC09NPF5.9 and BnaA09NPF5.10 were found preferential high expression in aged leaves and silique walls and nearly mature seeds. BnaC01NPF7.5 and BnaA01NPF7.6 showed higher expression at later development stages of seed, and BnaC07NPF8.7, BnaA06NPF8.8 and BnaC09NPF8.9 were preferentially higher expressed in aged leaves and silique wall. 


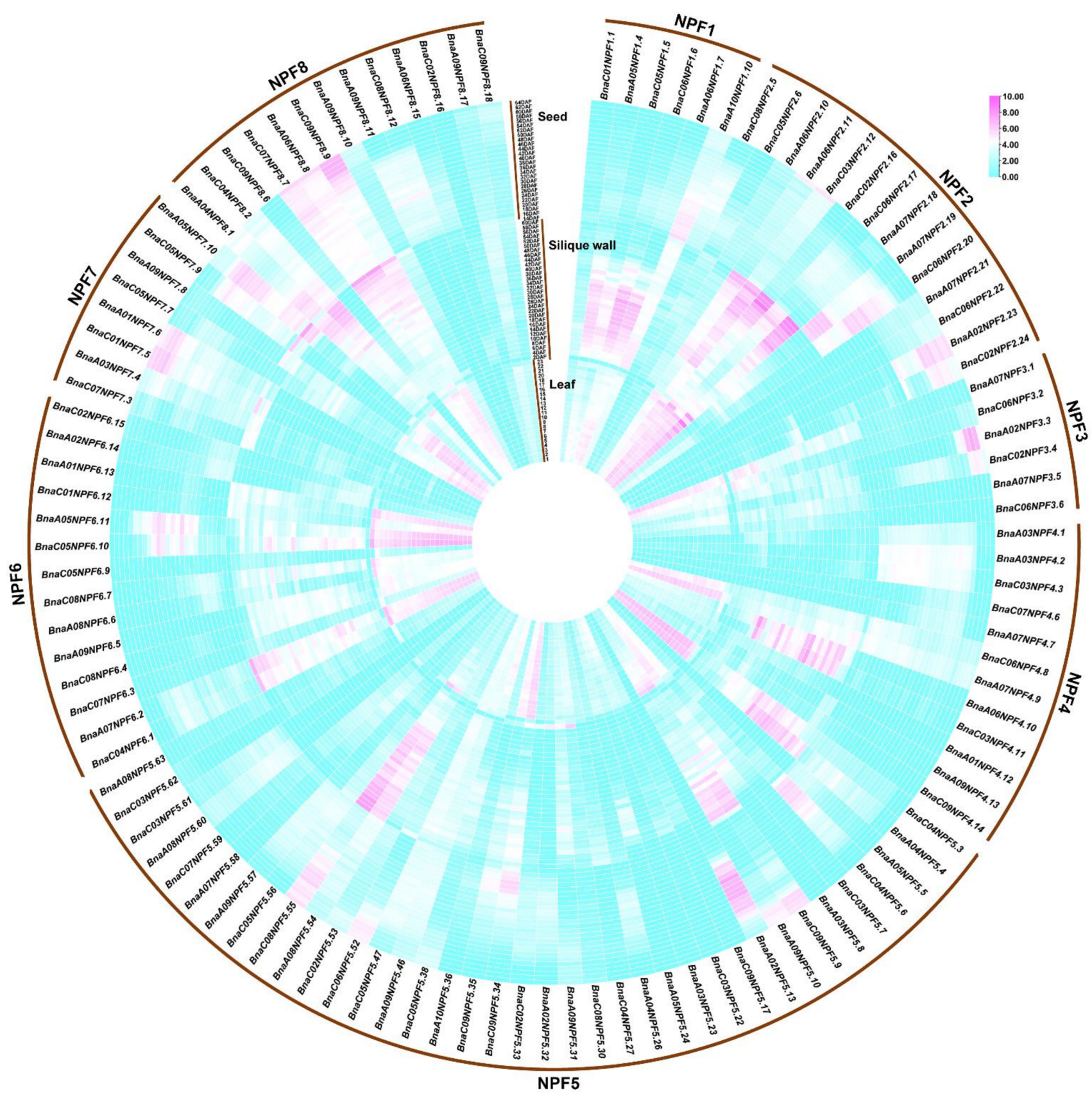

Figure 4. Expression changes of the BnaNPF genes at different development stages of leaves, silique wall and seeds. FPKM values were processed with $\log 2$ normalization on the column scale. The color scale represents relative expression levels from high (purple) to low (cyan).

\subsection{Expression Dynamic of NPF Genes during the Growth of B. napus under Vernalization}

There are differences in nutrition utilization and phytohormone distribution at different stages of plant growth. In order to explore the function and expression variation of BnaNPF genes, the expression trend of BnaNPF genes in leaves was analyzed during the growth of $B$. napus based on ZS11 transcription data from online data resources BnPIR (http:/ / cbi.hzau.edu.cn/bnapus/, Accessed on 4 May 2021). Although the expression levels were quite different, members of the same subfamily usually have the same expression trend in leaves during the growth of $B$. napus (Figure 5). The members in subfamily NPF1, NPF4, NPF5 and NPF7 seem to be the same expression trend, decline at beginning of vernalization or in the early stage of vernalization and rise after vernalization. For example, BnaC06NPF1.6, as an ortholog of AtNPF1.2 that was able to transport GA and JA, has the ex- 
act same expression trend and high expression level with BnaC04NPF5.3 (homologous with AtNPF5.1 that was able to transport GA, JA and ABA), which indicated that they might play important roles in phytohormone transport for a developmental phase transition. Some other members in NPF2, NPF3 and NPF6 shared this similar expression trend-that is, the expression level rising during vernalization and declining after vernalization. In typical cases, the expression level of BnaC02NPF2.6, BnaC06NPF3.2 and BnaC05NPF6.10 are dramatically raised from $\mathrm{T} 1$ to $\mathrm{T} 2$, and then begin to decline, which indicated that these members played an important role in the development stage during vernalization. Many BnaNPF genes showed diverse expression levels in the leaves of different cultivars at certain development stages (Figure S4). For example, BnaA05NPF1.4 and BnaC05NPF1.5 have no expression or lower expression levels in Shengli than other cultivars at T3 and T4 stages. At the T2 stage, BnaC02NPF2.16 showed obviously a higher expression level in cultivars Quinta, Shengli and Tapidor than others. BnaA06NPF8.8 has almost no expression during the whole development process in the three cultivars Shengli, Tapidor and Westar in comparison to other cultivars. These expression variations might lead to differences in nitrogen utilization efficiency, peptide transport and polar transport of phytohormone among the cultivars.
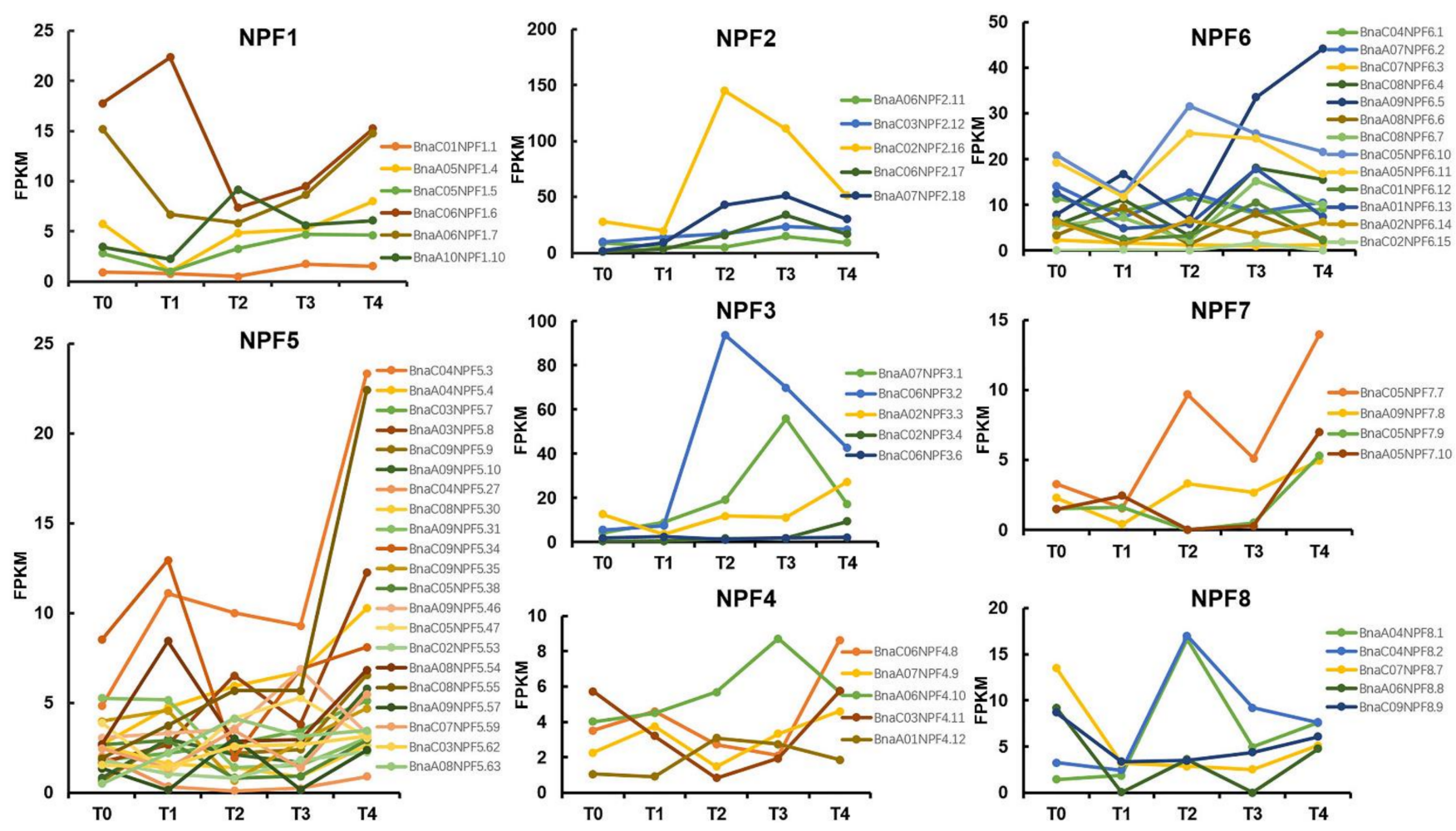

Figure 5. The expression changes of BnaNPF genes in leaves of ZS11 cultivar at five growth stages during vernalization. Plot FPKM value on the vertical $Y$-axis against growth stage on the horizontal $x$-axis. T0: 24 days post sowing and before vernalization; T1: 54 days post sowing and during vernalization; T2: 82 days post sowing and during vernalization; T3: 115 days post sowing and during vernalization; T4: 147 days post sowing and post vernalization.

\subsection{Transcriptional Analysis of BnaNPF Genes under Nitrate Deficiency}

Nitrate is the main substrate that NPF proteins transport, and more than one-third of NPF members have been reported to have a nitrate transport function in Arabidopsis [8]. Here, we analyzed the expression changes of BnaNPF genes under the condition of nitrogen suitability and deficiency. A total of 20 BnaNPF genes were detected to have relatively high expression and showed significant expression changes in shoot and/or root (Figure 6). Among them, six BnaNPF genes (BnaC06NPF4.16, BnaC04NPF6.1, BnaA07NPF6.2, BnaA08NPF6.6, BnaC05NPF7.7 and BnaA09NPF7.8) were expressed at a 
high level in both shoot and root, and the expression levels were significantly elevated in both shoot and root after being treated with low nitrogen. Ten BnaNPF genes were specifically expressed in root, of which seven (BnaA06NPF2.7, BnaC06NPF2.20, BnaC08NPF6.4, BnaA09NPF6.5, BnaA06NPF6.8, BnaC05NPF6.9 and BnaA05NPF7.10) were induced to highly express after low nitrogen treatment, which suggested they have a positive function for nitrogen absorption by roots. However, the expressions of the other three BnaNPF genes (BnaC06NPF4.8, BnaC09NPF4.14 and BnaC07NPF7.3) that specifically expressed in roots were declined under low nitrogen treatment. In addition, four BnaNPF genes that were specifically expressed in shoots also showed different expression changes under low nitrogen treatment: BnaC02NPF2.16 and BnaA06NPF4.10 were upregulated, and the other two (BnaC06NPF3.2 and BnaC07NPF6.3) declined after treated by low nitrogen.
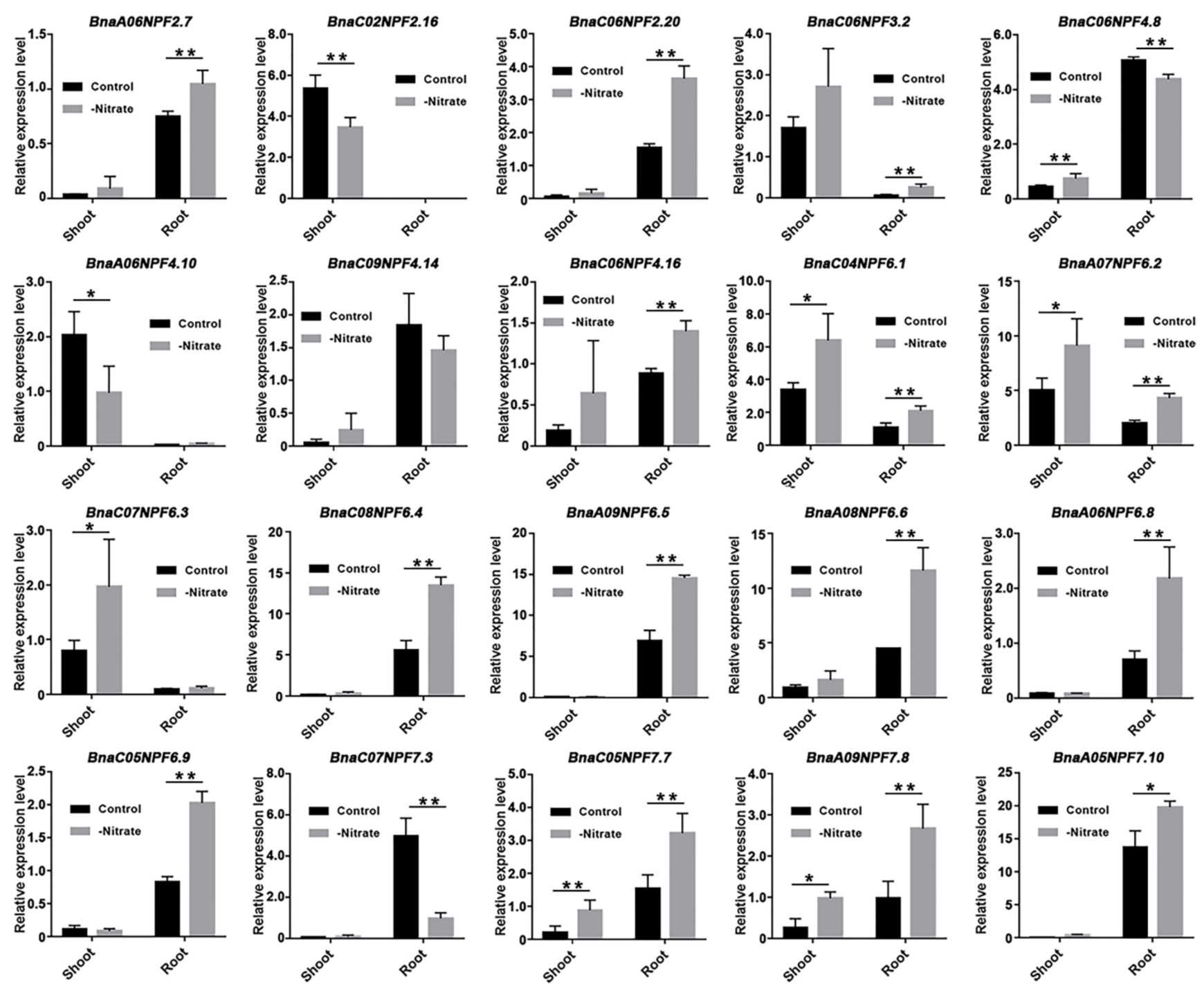

Figure 6. The expression changes detected for 20 BnaNPF genes under the condition of nitrogen suitability and deficiency. "Control" and "-Nitrate" represent treatments under nitrogen suitability and deficiency, respectively. "**and "***" represent the significance level of 0.05 and 0.01 , respectively.

\section{Discussion}

Although its genome is not the largest in comparison to the genomes of 33 plant species displayed in our study, B. napus contained the most NPF genes (Table 1). A total of 169 BnaNPF genes coding 186 proteins were identified in the B. napus genome 
in this study and designated as BnaNPF1.1 to BnaNPF8.19 in eight subfamilies based on phylogenetic analysis, and they exhibited evolutionary and syntenic relationships with NPF genes in Arabidopsis, B. rapa, and B. olereaca. Furthermore, the expression profiles of $B n a N P F$ genes in 90 diverse tissues, as well as expression changes at different development stages under vernalization between eight rapeseed cultivars and under nitrate deficiency, were determined. This study provides a piece of basic information for further functional characterization of BnaNPF genes in the growth and development of B. napus. Recently, in apple and wheat [13,39], the NPF protein family was characterized and also defined as eight subfamilies, and according to eight subfamilies of NPF defined in these species and phylogenetic analysis, the 169 BnaNPF genes were identified and classified into eight unambiguous subfamilies, and BnaNPF subfamilies showed similar member proportions with these in Arabidopsis and wheat [1,13]. Recently, the identification of NPF genes had been reported in B. napus; 193 and 199 BnaNPF genes were identified in two studies, respectively $[45,46]$. Here, in order to excavate functional NPF, we sifted out the candidate NPF protein with less than 200 amino acid residues and 20\% of the PTR2 domain missing, and only 169 NPF genes encoding 186 potential functional proteins were obtained for rapeseed finally. The 169 NPF proteins have a relatively complete protein sequence and PTR2 domain, and were likely to be functional and able to transport of substrates including amino acids, nitrate, phytohormones and glucosinolates. Brassicaceae species experienced a common whole genome soon after divergence from the Arabidopsis lineage approximately 17 to 20 million years ago $[40,48]$. B. napus is an allotetraploid (AnAnCnCn) that evolved from a spontaneous hybridization event between $B$. rape (ArAr) and B. oleracea $(\mathrm{CoCo})$ about 7500 years ago [42], and has suffered a whole-genome triplication and a hybridization event compared with Arabidopsis. In theory, there should be three times as much NPF genes in B. rapa and B. oleracea $(53 \times 3)$, and six times as much NPF genes in B. napus $(53 \times 3 \times 2)$ as in Arabidopsis. Fifty-three NPF members were identified in the Arabidopsis genome, it was expected that NPF genes may be expanded to about 160 genes in B. rapa or B. oleracea, and about 320 genes in B. napus genomes, respectively. However, only 82, 70 and 169 $B n a N P F$ genes were identified in these three species, respectively, in this study (Table 1), which revealed that genome replication was accompanied by a large-scale loss of genes during evolution that was identical to the previous reports $[49,50]$. The A sub-genome and $C$ sub-genome of B. napus (AACC) originated from B. rapa (AA) and B. oleracea (CC), respectively. Compared to both ancestral species, fewer NPF genes (76) were identified in the A sub-genome and more NPF genes (93) were discovered in the C sub-genome of B. napus. NPF genes in the $C$ sub-genome were amplified obviously, which happened probably due to chromosome rearrangement or gene replication when $B$. napus formed by hybridization between $B$. rapa and B. oleracea about 7500 years ago [42]. Besides, NPF genes distributed on B. rapa $(88.16 \%)$ and B. oleracea $(55.91 \%)$ genome keep good collinearity with $N P F$ genes on the $A$ and $C$ sub-genome of $B$. napus, respectively (Figure 1). These results indicate that $B n a N P F$ genes have undergone not only chromosome segment replication, but complex recombination and gene loss in evolution processes, which is consistent with the recent reports $[45,46]$.

The function and expression level of a gene is usually closely related to its gene characteristic and CREs [51]. Therefore, BnaNPF genes were further characterized for gene structures, protein conserved domains and CREs in this study. Most of the BnaNPF genes exhibited relatively concentrated distributional property in gene length (246-1568 $\mathrm{bp}$ ) and amino acid number (400-600). In gene structure, most of the BnaNPF genes contained at most six extrons, and different $B n a N P F$ subfamily genes exhibited significant exon-intron structural divergences, but BnaNPF within the same branches share similar gene structures, motifs, and localization patterns. Besides, CREs involved in hormone responses and MYB recognition site were detected in the promoter region of BnaNPF genes except for the common CREs, which indicated the expression of BnaNPF genes regulated by phytohormones and secondary metabolites (Figure 2). 
Gene expression patterns provided imperative clues to map out gene functionality. In this study, the expression level of BnaNPF genes was investigated in diverse tissues or organs of B. napus using the released transcriptome information resource (http:/ / yanglab. hzau.edu.cn/BnTIR/eFP, Accessed on 4 May 2021). Gene expression analysis showed that BnaNPF genes have significantly different and complex expression patterns across different subfamilies, but the expression pattern was conserved in each clade within a subfamily, which reflected structure and function uniform (Figure 3). Some BnaNPF genes showed obvious tissue preferential expression. Half of the BnaNPF1 genes having ultrahigh expression levels in silique wall at the early and middle development stages of silique indicated efficient nitrogen transport for nutrient synthesis in the seed. BnaA07NPF2.18, orthologous to AtNPF2.13/NRT1.7 that was able to transport nitrate, glucosinolates, JAs and GAs [14,52], was expressed at an ultrahigh expression level in the bud, pollen, filament and petal, which contribute to the previous reports that nitrate and nitrogen regulated flowering, and high nitrate/nitrogen helped promote flowering [53], and phytohormone played an important role in the regulation of flower organogenesis [54,55].

NPF proteins can transport a huge variety of substrates, including dipeptides, nitrate, glucosinolates, amino acids and several plant hormones [8], and the complex gene expression pattern would endow BnaNPF versatile roles in the growth and development of B. napus. Many BnaNPF genes were found to have a changing expression in the development of leaf, silique wall and seed that played a key role in yield (Figure 4). For example, in the BnaNPF2 subfamily, BnaA06NPF2.10, BnaA06NPF2.11 and BnaC03NPF2.12 were orthologs to AtNPF2.10, and BnaC02NPF2.16, BnaC06NPF2.17 and BnaA07NPF2.18 were orthologs to AtNPF2.13, of which two Arabidopsis orthologs had the function of transporting glucosinolates $[11,56]$ and showed up-regulated expression in the later stages of the silique and seed development of $B$. napus. Many CREs involved in hormone responses were detected in the promoter region of BnaNPF genes, including IAA- (103/169 genes), ABA- (123/169 genes), and MeJA-responsive CRE (119/169 genes) (Figure 2 and Table S4), which suggested their potential hormone-inducing characteristics. The process of plant growth and development was regulated by phytohormone directly, which might be why the transcription of BnaNPF genes was regulated responding to growth and development. Besides, with the development of plant organs, secondary metabolite accumulation level perhaps also played a part in the expression changes of some BnaNPF genes because of the existence of the MYB recognition site in their promoters [57].

The expression changes of BnaNPF genes during the growth of B. napus under natural vernalization were analyzed in this study. Vernalization is an important process that regulates the transition from vegetative growth to reproduction in B. napus [58,59], and involved in the regulation of various environmental factors and hormones [60]. The BnaNPF genes that expressed in leaves exhibited two expression trends: the first one, decline at the beginning of vernalization or in the early stage of vernalization and a rise after vernalization (most of the members of the NPF1, NPF4, NPF5 and NPF7 subfamily); the second one, the expression level was raised during vernalization and declined after vernalization (most of the members of the NPF2, NPF3 and NPF6 subfamily) (Figure 5). These results indicate that many BnaNPF genes might participate in floral transition and play different roles in the reproduction and development of $B$. napus. Based on the transcriptome data of eight cultivars from the BnPIR database, significant expression variation was found for some BnaNPF genes in different cultivars (Figure S4). These expression variations might lead to differences in transport of the corresponding substrates among the cultivars, which is expected for further functional research in the future.

Nitrate and phytohormone signaling pathways crosstalk to modulate growth and developmental programs in a multifactorial manner [61]. So far, more than half of the functionally characterized NPF genes have been demonstrated to be able to transport nitrate in Arabidopsis [13]. Here, twenty BnaNPF genes, ortholog to 11 AtNPF genes, were detected to respond to low nitrate treatment (Figure 6). The six members of the BnaNPF6 subfamily, BnaNPF6.4-6.9 orthologous with AtNPF6.3/NRT1.1, were predominantly expressed in roots 
and were significantly up-regulated under low nitrogen treatment, suggesting their functional importance in nitrogen utilization efficiency. AtNPF6.3 is the first plant NPF member that is characterized for functioning in nitrate uptake in the root, root-to-shoot transport and transceptor in sensing/signaling, and govern many molecular, physiological, and morphological responses to nitrate $[6,15,16]$. The gene expansion and consistent expression patterns in B. napus indicated the function uniformity of NPF6.3 orthologs in nitrogen utilization efficiency as previously reported [62,63]. As the ortholog of AtNPF2.9/NRT1.9 has been reported to facilitate the loading of nitrate into the root phloem and enhance downward nitrate transport in roots [64], BnaA06NPF2.7 was up-regulated significantly in root under low nitrate conditions, while its homolog in rice, OsNPF2.4, was discovered by a genome-wide association study (GWAS) on nitrogen utilization efficiency-related agronomic traits [65]. So, BnaA06NPF2.7 might also play an important role in nitrate transport in roots in B. napus, which needs to be characterized in the future. In addition to the role as a nutrient, nitrate acts as a signal molecular, and $\mathrm{N}$ nutrition and plant hormone signaling pathways are closely interconnected [61]. BnaC02NPF2.16 and BnaC06NPF3.2, orthologues with AtNPF2.13 and AtNPF3.1, respectively, were predominantly expressed in leaves. According to previous reports regarding AtNPF2.13 and AtNPF3.1, remobilizing nitrate from old to young leaves involved GA accumulation and responses [66-68]. BnaC02NPF2.16 and BnaC06NPF3.2 might function in nitrite accumulation in leaves coupling hormone signal, which may be possible but needs to be verified in the future.

In this study, we provided a comprehensive understanding of the evolution and expression characteristics of BnaNPF genes in B. napus. It gives an important implication for further understanding the biological functions of individual BnaNPF genes. However, the study only provided a preliminary characterization of BnaNPF genes, and large functional validation work needs to be done in further work to understand the roles of BnaNPF genes.

\section{Materials and Methods}

\subsection{Data Resource Related to NPF Gene Acquisition}

The 53 NPF protein sequences from Arabidopsis were used as query, and a BLASTp search (E-value $<10^{-10}$ ) was performed to identify NPF genes in B. rapa, B. oleracea, B. napus and other plant species through the online BLAST tool in the databases, including The Arabidopsis Information Resource (TAIR, https:/ / www.Arabidopsis.org/, Genome Version Araport11, Accessed on 4 May 2021), the Brassica Database (BRAD, http:/ / brassicadb.org/ brad/, var. 'Chiifu' and 'TO1000', Accessed on 4 May 2021 ), Brassica napus Pan-Genome Information Resource (BnPIR, http:/ / cbi.hzau.edu.cn/bnapus/, var. ZS11, Accessed on 4 May 2021), and National Center for Biotechnology Information (NCBI, https:/ /blast.ncbi. nlm.nih.gov / Blast.cgi, Accessed on 4 May 2021). Then, the potential NPF proteins were confirmed through the hidden Markov model (HMM) search program (HMMER v3.0, http:/ /hmmer.janelia.org/, Accessed on 4 May 2021) with E-value below e-200, and the conserved domain database (CDD) (http:/ / www.ncbi.nlm.nih.gov/Structure/bwrpsb / bwrpsb.cgi, Accessed on 4 May 2021) based on the presence of the PTR2 domains (PF00854). Furthermore, the candidate NPF protein of B. rapa, B. oleracea and B. napus with less than 200 amino acid residues and $20 \%$ of PTR2 domains missing was removed, and the rest were thought to be considered to be functional and used for further analysis.

\subsection{Multiple Sequence Alignment and BnaNPF Genes Nomenclature}

Full-length sequences of the NPF proteins from Arabidopsis and three Brassica crops were aligned with ClustalW, and then these alignments were used to construct the phylogenetic trees by the software MEGA Version 10.1.0 [69] with the neighbor-joining method. P-distance, pairwise deletion, and bootstrapping (1000 replicates) were set as the required parameters.

NPF genes were named according to the nomenclature Leran et al. (2014) recommended [1]. According to eight subfamilies of NPF in Arabidopsis and phylogenetic relationships, the clade number of NPF members would be ensured. Then, NPF members 
were named with two or three letters to identify the species, followed by "NPF + clade number (followed by a point) + the order number (which they are identified in phylogenetic tree)", for instance, "BraNPF2.3". Consequently, this second number is used to differentiate genes within the species and does not reflect orthologous relationships. The NPF members from $B$. napus obeyed the nomenclature convention but modified with adding chromosome between species name and "NPF". If multiple NPF proteins were translated from the transcripts of the same gene, they were distinguished by the English letters " $a$ ", " $b$ " and " $c$ ".

\subsection{Chromosomal Location and Syntenic Analysis}

The genomic locations of all BnaNPF genes were mapped to chromosomes of the B. napus genome according to the reference genome information of ZS11 in the BnPIR database. The synteny orthologous gene pairs were identified based on BLASTP (identity $>75 \%$, and E-value $<10^{-10}$ ) and phylogenetic relationship. The chromosomal regions within $200 \mathrm{~kb}$ containing a string of two or more genes were defined as tandem duplication [70]. The nonsynonymous rate $(\mathrm{Ka})$, synonymous rate $(\mathrm{Ks})$, and $\mathrm{Ka} / \mathrm{Ks}$ between the orthologous gene pairs were calculated using the NY method implemented in the $\mathrm{Ka} / \mathrm{Ks}$ calculator program [71] according to gene CDS pairwise alignment performed with Clustal W (https:/ / www.genome.jp/tools-bin/clustalw, Accessed on 4 May 2021).

\subsection{Functional Domain Validation and Cis-Acting Regulatory Elements (CREs) Prediction}

The protein sequence and full-length coding sequences (CDS) information of the AtNPFs and BnaNPFs were retrieved and extracted from the Arabidopsis Information Resource (TAIR: https: / / www.Arabidopsis.org/index.jsp, Accessed on 4 May 2021) and Brassica napus pan-genome information resource (BnPIR: http:/ / cbi.hzau.edu.cn/bnapus / index.php, Accessed on 4 May 2021). To examine the structural divergence among the NPF proteins in Arabidopsis and B. napus, the protein sequences were subjected to the HMMER software (http: / / www.ebi.ac.uk/Tools/hmmer, Accessed on 4 May 2021) to predict and characterize the conserved domains with default parameters. A $2.0 \mathrm{~kb}$ genomic sequence upstream from the start codon was downloaded for each gene from the BnPIR website (http: / / cbi.hzau.edu.cn/bnapus/index.php, Accessed on 4 May 2021). These sequences were subjected to plantCARE (http:/ / bioinformatics.psb.ugent.be/webtools/plantcare/html/, Accessed on 4 May 2021) to identify putative CREs, and CRE distribution in the promoter region was displayed by TBtools software [72]. The Gene Structure Display Server (GSDS Version 2.0, http:/ / gsds.cbi.pku.edu.cn/index.php, Accessed on 4 May 2021) was used to display the exon-intron structures of the NPFs in Arabidopsis and B. napus.

\subsection{Identification of the Expression Pattern of BnaNPF Genes in B. napus}

The fragments per kilobase of exon model per million mapped fragments (FPKM) value of 169 BnaNPF genes in different organs or tissues, including cotyledon, root, vegetative rosette, stem peel (peel of upper, middle and lower stem), leaves (23 parts or periods), sepal, petal, filament, pollen, bud, silique wall (30 development periods) and seeds (24 development periods) based on transcriptome information, were retrieved from BnTIR (http: / /yanglab.hzau.edu.cn/BnTIR/eFP, Accessed on 4 May 2021). RNA-seq for leaves of all eight rapeseed accessions at five stages with a one-month interval derived from BnPIR (http: / / cbi.hzau.edu.cn/bnapus/expression/, Accessed on 4 May 2021), including one stage before vernalization (T0), three stages during vernalization (T1, T2 and T3), and one stage post vernalization (T4), was used to analyze BnaNPF gene expression patterns at different development stages.

\subsection{Expression Analysis of BnaNPF Genes under Low Nitrate Stress}

To further investigate the transcriptional responses of BnaNPF genes under low nitrate stress, the uniform B. napus seedlings (var. ZS11) were hydroponically cultured in Hoagland nutrient solution for 10 days at 7 days after seed germination, and then parts of them were 
transferred to Hoagland nutrient solution modified with low nitrate $\left(0.3 \mathrm{mM} \mathrm{NO}^{3-}\right)$ for 3 days. The rapeseed seedlings were cultivated in the culture room as Cui et al. (2020) described [73]. The shoots and roots under low nitrogen treatment for $72 \mathrm{~h}$ and control were individually harvested and immediately stored at $-80{ }^{\circ} \mathrm{C}$ for RNA isolation, and each sample contained 3 independent biological replicates. Total RNA was isolated from the frozen samples using a RNAprep Pure Plant Kit (Tiangen), and first-strand cDNA was synthesized from the total RNA using a PrimeScriptTM RT Master Mix Kit (TaKaRa). The cDNA was subjected to quantitative analysis using SYBR ${ }^{\circledR}$ Premix Ex Taq ${ }^{\mathrm{TM}}$ (Takara Bio, Shiga, Japan) on the Applied Biosystems StepOne ${ }^{\mathrm{TM}}$ Plus Real-time PCR System (Thermo Fisher Scientific, Waltham, MA, USA), as previously described [73]. The BnaNPF primer sequences were obtained from the qPCR Primer Database [74] and are listed in Table S1. The housekeeping gene BnaACTIN7 was used as a reference gene for normalization and to analyze the BnaNPF gene expression levels via the $2^{-\Delta \Delta C t}$ method. Three independent technical replicates were performed for each sample.

\section{Conclusions}

A total of 169 NPF gene members were identified in the B. napus genome and classified into eight subfamilies in this study. The BnaNPF genes were unevenly distributed in the $B$. napus genome and exhibited obvious synteny and orthologous duplication with NPF genes in Arabidopsis, B. rapa and B. olereaca. Moreover, the complex expression patterns of NPF genes in various tissues and periods were investigated, and the expression changes at different development stages under nature vernalization and response to nitrate deficiency were determined in B. napus. The evolution and expression pattern analysis of NPF genes will provide valuable information for further functional characterization in rapeseed.

Supplementary Materials: The following are available online at https:/ / www.mdpi.com/article / 10.3390 /ijms22094944/s1, Figure S1: Phylogenetic relationships of the NPF proteins in Arabidopsis and B. napus. Neighbour-joining bootstrap values are shown above the branch, the branches of different subfamilies are colored differently, and the terminals of the branch labeled red represent the Arabidopsis NPF proteins. Figure S2: The synteny of NPF genes among Arabidopsis, B. rapa, B. oleracea and B. napus. Figure S3: Gene structure and protein conserved domain architecture of the BnaNPF genes. On the left is the gene structure and on the right is the protein conserved domain architecture. NPF1 and NPF2 clustered in a branch were displayed in (a), NPF3, NPF4 and NPF6 were displayed in (b), NPF5 was displayed in (c), and NPF7 and NPF8 clustered in a branch were displayed in (d). Figure S4: The expression diversity of BnaNPF genes in leaves of eight cultivars at five growth stages during vernalization. Table S1: The primer sequences for the qRT-PCR. Table S2: Ka, Ks and Ka/Ks of orthologous pairs on BnaNPF and AtNPF genes. Table S3: The synteny relationship of NPF genes between B. napus and B. rape and B. oleracea. Table S4: The CREs detected in the promoter of the BnaNPF genes.

Author Contributions: Conceptualization, M.L. and J.H.; methodology, H.C. and J.H.; software, J.H. and Q.C.; validation, H.C. and W.Z.; formal analysis, Q.C.; investigation, Q.C. and H.F.; resources, H.C.; data curation, Y.H.; writing—original draft preparation, H.C.; writing—review and editing, M.L. and J.H.; visualization, H.C.; supervision, and H.F.; project administration, H.C.; funding acquisition, H.C. and W.Z. All authors have read and agreed to the published version of the manuscript.

Funding: This research was funded by the Key Research Plan Project of Shaanxi Province (grant number 2020ZDLNY04-01), the Chinese Postdoctoral Science Foundation (grant number 2019M662539), the Key Scientific Research Projects of Colleges and Universities in Henan Province (grant number K20A180031) and the Postdoctoral Research Grant of Henan Province (grant number 201901004).

Institutional Review Board Statement: Not applicable.

Informed Consent Statement: Not applicable.

Data Availability Statement: Not applicable

Acknowledgments: Not applicable.

Conflicts of Interest: The authors declare no conflict of interest. 


\section{References}

1. Leran, S.; Varala, K.; Boyer, J.C.; Chiurazzi, M.; Crawford, N.; Daniel-Vedele, F.; David, L.; Dickstein, R.; Fernandez, E.; Forde, B.; et al. A Unified Nomenclature of Nitrate Transporter 1/Peptide Transporter Family Members in Plants. Trends. Plant. Sci. 2014, 19, 5-9. [CrossRef] [PubMed]

2. Daniel, H.; Spanier, B.; Kottra, G.; Weitz, D. From Bacteria to Man: Archaic Proton-Dependent Peptide Transporters at Work. Physiology (Bethesda) 2006, 21, 93-102. [CrossRef] [PubMed]

3. Meredith, D. Review. The Mammalian Proton-Coupled Peptide Cotransporter PEPT1: Sitting on the Transporter-Channel Fence? Philos. Trans. R. Soc. Lond. B. Biol. Sci. 2009, 364, 203-207. [CrossRef] [PubMed]

4. Tsay, Y.F.; Schroeder, J.I.; Feldmann, K.A.; Crawford, N.M. The Herbicide Sensitivity Gene Chl1 of Arabidopsis Encodes a Nitrate-Inducible Nitrate Transporter. Cell 1993, 72, 705-713. [CrossRef]

5. Tsay, Y.F. Plant Science How to Switch Affinity. Nature 2014, 507, 44-45. [CrossRef]

6. Sun, J.; Zheng, N. Molecular Mechanism Underlying the Plant NRT1.1 Dual-Affinity Nitrate Transporter. Front. Physiol. 2015, 6, 386. [CrossRef]

7. Tegeder, M.; Rentsch, D. Uptake and Partitioning of Amino Acids and Peptides. Mol. Plant 2010, 3, 997-1011. [CrossRef]

8. Corratge-Faillie, C.; Lacombe, B. Substrate (Un)Specificity of Arabidopsis NRT1/PTR Family (NPF) Proteins. J. Exp. Bot. 2017, 68, 3107-3113. [CrossRef]

9. Krouk, G.; Lacombe, B.; Bielach, A.; Perrine-Walker, F.; Malinska, K.; Mounier, E.; Hoyerova, K.; Tillard, P.; Leon, S.; Ljung, K.; et al. Nitrate-Regulated Auxin Transport by NRT1.1 Defines a Mechanism for Nutrient Sensing in Plants. Dev. Cell. 2010, 18, 927-937. [CrossRef]

10. Kanno, Y.; Hanada, A.; Chiba, Y.; Ichikawa, T.; Nakazawa, M.; Matsui, M.; Koshiba, T.; Kamiya, Y.; Seo, M. Identification of an Abscisic Acid Transporter by Functional Screening Using the Receptor Complex as a Sensor. Proc. Natl. Acad. Sci. USA 2012, 109, 9653-9658. [CrossRef]

11. Nour-Eldin, H.H.; Andersen, T.G.; Burow, M.; Madsen, S.R.; Jorgensen, M.E.; Olsen, C.E.; Dreyer, I.; Hedrich, R.; Geiger, D.; Halkier, B.A. NRT/PTR Transporters Are Essential for Translocation of Glucosinolate Defence Compounds to Seeds. Nature 2012, 488, 531-534. [CrossRef]

12. Boursiac, Y.; Leran, S.; Corratge-Faillie, C.; Gojon, A.; Krouk, G.; Lacombe, B. ABA Transport and Transporters. Trends Plant Sci. 2013, 18, 325-333. [CrossRef]

13. Wang, H.; Wan, Y.; Buchner, P.; King, R.; Ma, H.; Hawkesford, M.J. Phylogeny and Gene Expression of the Complete Nitrate Transporter 1/Peptide Transporter Family in Triticum aestivum. J. Exp. Bot. 2020, 71, 4531-4546. [CrossRef]

14. Chiba, Y.; Shimizu, T.; Miyakawa, S.; Kanno, Y.; Koshiba, T.; Kamiya, Y.; Seo, M. Identification of Arabidopsis thaliana NRT1/PTR Family (NPF) Proteins Capable of Transporting Plant Hormones. J. Plant. Res. 2015, 128, 679-686. [CrossRef]

15. Krouk, G.; Crawford, N.M.; Coruzzi, G.M.; Tsay, Y.F. Nitrate Signaling: Adaptation to Fluctuating Environments. Curr. Opin. Plant. Biol. 2010, 13, 266-273. [CrossRef]

16. Bouguyon, E.; Brun, F.; Meynard, D.; Kubes, M.; Pervent, M.; Leran, S.; Lacombe, B.; Krouk, G.; Guiderdoni, E.; Zazimalova, E.; et al. Multiple Mechanisms of Nitrate Sensing by Arabidopsis Nitrate Transceptor NRT1.1. Nat. Plants 2015, 1, 15015. [CrossRef]

17. Chiang, C.S.; Stacey, G.; Tsay, Y.F. Mechanisms and Functional Properties of Two Peptide Transporters, AtPTR2 and FPTR2. J. Biol. Chem. 2004, 279, 30150-30157. [CrossRef]

18. Hammes, U.Z.; Meier, S.; Dietrich, D.; Ward, J.M.; Rentsch, D. Functional Properties of the Arabidopsis Peptide Transporters AtPTR1 and AtPTR5. J. Biol. Chem. 2010, 285, 39710-39717. [CrossRef]

19. Yendrek, C.R.; Lee, Y.C.; Morris, V.; Liang, Y.; Pislariu, C.I.; Burkart, G.; Meckfessel, M.H.; Salehin, M.; Kessler, H.; Wessler, H.; et al. A Putative Transporter Is Essential for Integrating Nutrient and Hormone Signaling with Lateral Root Growth and Nodule Development in Medicago truncatula. Plant. J. 2010, 62, 100-112. [CrossRef]

20. Bagchi, R.; Salehin, M.; Adeyemo, O.S.; Salazar, C.; Shulaev, V.; Sherrier, D.J.; Dickstein, R. Functional Assessment of the Medicago Truncatula NIP/LATD Protein Demonstrates That It Is a High-Affinity Nitrate Transporter. Plant. Physiol. 2012, 160, 906-916. [CrossRef]

21. Salehin, M.; Huang, Y.S.; Bagchi, R.; Sherrier, D.J.; Dickstein, R. Allelic Differences in Medicago Truncatula Nip/Latd Mutants Correlate with Their Encoded Proteins' Transport Activities in Planta. Plant. Signal. Behav. 2013, 8, e22813. [CrossRef] [PubMed]

22. Hsu, P.K.; Tsay, Y.F. Two Phloem Nitrate Transporters, NRT1.11 and NRT1.12, Are Important for Redistributing Xylem-Borne Nitrate to Enhance Plant Growth. Plant. Physiol. 2013, 163, 844-856. [CrossRef] [PubMed]

23. Taochy, C.; Gaillard, I.; Ipotesi, E.; Oomen, R.; Leonhardt, N.; Zimmermann, S.; Peltier, J.B.; Szponarski, W.; Simonneau, T.; Sentenac, H.; et al. The Arabidopsis Root Stele Transporter NPF2.3 Contributes to Nitrate Translocation to Shoots under Salt Stress. Plant. J. 2015, 83, 466-479. [CrossRef] [PubMed]

24. Almagro, A.; Lin, S.H.; Tsay, Y.F. Characterization of the Arabidopsis Nitrate Transporter NRT1.6 Reveals a Role of Nitrate in Early Embryo Development. Plant. Cell 2008, 20, 3289-3299. [CrossRef]

25. Sugiura, M.; Georgescu, M.N.; Takahashi, M. A Nitrite Transporter Associated with Nitrite Uptake by Higher Plant Chloroplasts. Plant. Cell Physiol. 2007, 48, 1022-1035. [CrossRef]

26. Karim, S.; Holmstrom, K.O.; Mandal, A.; Dahl, P.; Hohmann, S.; Brader, G.; Palva, E.T.; Pirhonen, M. AtPTR3, a WoundInduced Peptide Transporter Needed for Defence against Virulent Bacterial Pathogens in Arabidopsis. Planta 2007, 225, 1431-1445. [CrossRef] 
27. Leran, S.; Edel, K.H.; Pervent, M.; Hashimoto, K.; Corratge-Faillie, C.; Offenborn, J.N.; Tillard, P.; Gojon, A.; Kudla, J.; Lacombe, B. Nitrate Sensing and Uptake in Arabidopsis Are Enhanced by Abi2, a Phosphatase Inactivated by the Stress Hormone Abscisic Acid. Sci Signal. 2015, 8, ra43. [CrossRef]

28. Okamoto, M.; Vidmar, J.J.; Glass, A.D. Regulation of NRT1 and NRT2 Gene Families of Arabidopsis thaliana: Responses to Nitrate Provision. Plant. Cell Physiol. 2003, 44, 304-317. [CrossRef]

29. Chiu, C.C.; Lin, C.S.; Hsia, A.P.; Su, R.C.; Lin, H.L.; Tsay, Y.F. Mutation of a Nitrate Transporter, AtNRT1:4, Results in a Reduced Petiole Nitrate Content and Altered Leaf Development. Plant. Cell Physiol. 2004, 45, 1139-1148. [CrossRef]

30. Morere-Le Paven, M.C.; Viau, L.; Hamon, A.; Vandecasteele, C.; Pellizzaro, A.; Bourdin, C.; Laffont, C.; Lapied, B.; Lepetit, M.; Frugier, F.; et al. Characterization of a Dual-Affinity Nitrate Transporter MtNRT1.3 in the Model Legume Medicago truncatula. J. Exp. Bot. 2011, 62, 5595-5605. [CrossRef]

31. Li, J.Y.; Fu, Y.L.; Pike, S.M.; Bao, J.; Tian, W.; Zhang, Y.; Chen, C.Z.; Zhang, Y.; Li, H.M.; Huang, J.; et al. The Arabidopsis Nitrate Transporter NRT1.8 Functions in Nitrate Removal from the Xylem Sap and Mediates Cadmium Tolerance. Plant. Cell 2010, 22, 1633-1646. [CrossRef]

32. Chen, C.Z.; Lv, X.F.; Li, J.Y.; Yi, H.Y.; Gong, J.M. Arabidopsis NRT1.5 Is Another Essential Component in the Regulation of Nitrate Reallocation and Stress Tolerance. Plant. Physiol. 2012, 159, 1582-1590. [CrossRef]

33. Ouyang, J.; Cai, Z.Y.; Xia, K.F.; Wang, Y.Q.; Duan, J.; Zhang, M.Y. Identification and Analysis of Eight Peptide Transporter Homologs in Rice. Plant. Sci. 2010, 179, 374-382. [CrossRef]

34. Komarova, N.Y.; Thor, K.; Gubler, A.; Meier, S.; Dietrich, D.; Weichert, A.; Suter Grotemeyer, M.; Tegeder, M.; Rentsch, D. AtPTR1 and AtPTR5 Transport Dipeptides in Planta. Plant. Physiol. 2008, 148, 856-869. [CrossRef]

35. Choi, M.G.; Kim, E.J.; Song, J.Y.; Choi, S.B.; Cho, S.W.; Park, C.S.; Kang, C.S.; Park, Y.I. Peptide Transporter2 (PTR2) Enhances Water Uptake During Early Seed Germination in Arabidopsis thaliana. Plant. Mol. Biol. 2020, 102, 615-624. [CrossRef]

36. Pellizzaro, A.; Alibert, B.; Planchet, E.; Limami, A.M.; Morere-Le Paven, M.C. Nitrate Transporters: An Overview in Legumes. Planta 2017, 246, 585-595. [CrossRef]

37. Babst, B.A.; Gao, F.; Acosta-Gamboa, L.M.; Karve, A.; Schueller, M.J.; Lorence, A. Three NPF Genes in Arabidopsis Are Necessary for Normal Nitrogen Cycling under Low Nitrogen Stress. Plant. Physiol. Biochem. 2019, 143, 1-10. [CrossRef]

38. Bai, H.; Euring, D.; Volmer, K.; Janz, D.; Polle, A. The Nitrate Transporter (Nrt) Gene Family in Poplar. PLoS ONE 2013, 8, e72126. [CrossRef]

39. Wang, Q.; Liu, C.; Dong, Q.; Huang, D.; Li, C.; Li, P.; Ma, F. Genome-Wide Identification and Analysis of Apple Nitrate Transporter 1/Peptide Transporter Family (NPF) Genes Reveals MdNPF6.5 Confers High Capacity for Nitrogen Uptake under Low-Nitrogen Conditions. Int. J. Mol. Sci. 2018, 19, 2761. [CrossRef]

40. Lysak, M.A.; Koch, M.A.; Pecinka, A.; Schubert, I. Chromosome Triplication Found across the Tribe Brassiceae. Genome Res. 2005, 15, 516-525. [CrossRef]

41. Wang, X.; Wang, H.; Wang, J.; Sun, R.; Wu, J.; Liu, S.; Bai, Y.; Mun, J.H.; Bancroft, I.; Cheng, F.; et al. The Genome of the Mesopolyploid Crop Species Brassica rapa. Nat. Genet. 2011, 43, 1035-1039. [CrossRef] [PubMed]

42. Chalhoub, B.; Denoeud, F.; Liu, S.; Parkin, I.A.; Tang, H.; Wang, X.; Chiquet, J.; Belcram, H.; Tong, C.; Samans, B.; et al. Plant Genetics. Early Allopolyploid Evolution in the Post-Neolithic Brassica napus Oilseed Genome. Science 2014, 345, 950-953. [CrossRef] [PubMed]

43. Liu, S.; Liu, Y.; Yang, X.; Tong, C.; Edwards, D.; Parkin, I.A.; Zhao, M.; Ma, J.; Yu, J.; Huang, S.; et al. The Brassica oleracea Genome Reveals the Asymmetrical Evolution of Polyploid Genomes. Nat. Commun. 2014, 5, 3930. [CrossRef] [PubMed]

44. Sun, F.; Fan, G.; Hu, Q.; Zhou, Y.; Guan, M.; Tong, C.; Li, J.; Du, D.; Qi, C.; Jiang, L.; et al. The High-Quality Genome of Brassica napus Cultivar 'Zs11' Reveals the Introgression History in Semi-Winter Morphotype. Plant. J. 2017, 92, 452-468. [CrossRef]

45. Zhang, H.; Li, S.; Shi, M.; Wang, S.; Shi, L.; Xu, F.; Ding, G. Genome-Wide Systematic Characterization of the NPF Family Genes and Their Transcriptional Responses to Multiple Nutrient Stresses in Allotetraploid Rapeseed. Int. J. Mol. Sci. 2020, $21,5947$. [CrossRef]

46. Wen, J.; Li, P.F.; Ran, F.; Guo, P.C.; Zhu, J.T.; Yang, J.; Zhang, L.L.; Chen, P.; Li, J.N.; Du, H. Genome-Wide Characterization, Expression Analyses, and Functional Prediction of the NPF Family in Brassica napus. BMC Genomics 2020, 21, 871. [CrossRef]

47. Wittkopp, P.J.; Kalay, G. Cis-regulatory Elements: Molecular Mechanisms and Evolutionary Processes Underlying Divergence. Nat. Rev. Genet. 2011, 6, 59-69. [CrossRef]

48. Town, C.D.; Cheung, F.; Maiti, R.; Crabtree, J.; Haas, B.J.; Wortman, J.R.; Hine, E.E.; Althoff, R.; Arbogast, T.S.; Tallon, L.J.; et al. Comparative Genomics of Brassica Oleracea and Arabidopsis thaliana Reveal Gene Loss, Fragmentation, and Dispersal after Polyploidy. Plant. Cell 2006, 18, 1348-1359. [CrossRef]

49. De Bodt, S.; Maere, S.; Van de Peer, Y. Genome Duplication and the Origin of Angiosperms. Trends Ecol. Evol. 2005, 20, 591-597. [CrossRef]

50. Mun, J.H.; Kwon, S.J.; Yang, T.J.; Seol, Y.J.; Jin, M.; Kim, J.A.; Lim, M.H.; Kim, J.S.; Baek, S.; Choi, B.S.; et al. Genome-Wide Comparative Analysis of the Brassica rapa Gene Space Reveals Genome Shrinkage and Differential Loss of Duplicated Genes after Whole Genome Triplication. Genome Biol. 2009, 10, R111. [CrossRef]

51. Jeffares, D.C.; Penkett, C.J.; Bahler, J. Rapidly Regulated Genes Are Intron Poor. Trends Genet. 2008, 24, 375-378. [CrossRef]

52. Fan, S.C.; Lin, C.S.; Hsu, P.K.; Lin, S.H.; Tsay, Y.F. The Arabidopsis Nitrate Transporter NRT1.7, Expressed in Phloem, Is Responsible for Source-to-Sink Remobilization of Nitrate. Plant. Cell 2009, 21, 2750-2761. [CrossRef] 
53. Lin, Y.L.; Tsay, Y.F. Influence of Differing Nitrate and Nitrogen Availability on Flowering Control in Arabidopsis. J. Exp. Bot. 2017, 68, 2603-2609. [CrossRef] [PubMed]

54. Li, Q.; Liu, B. Genetic Regulation of Maize Flower Development and Sex Determination. Planta 2017, 245, 1-14. [CrossRef]

55. Fredes, I.; Moreno, S.; Diaz, F.P.; Gutierrez, R.A. Nitrate Signaling and the Control of Arabidopsis Growth and Development. Curr. Opin. Plant. Biol. 2019, 47, 112-118. [CrossRef]

56. Andersen, T.G.; Nour-Eldin, H.H.; Fuller, V.L.; Olsen, C.E.; Burow, M.; Halkier, B.A. Integration of Biosynthesis and Long-Distance Transport Establish Organ-Specific Glucosinolate Profiles in Vegetative Arabidopsis. Plant. Cell 2013, 25, 3133-3145. [CrossRef]

57. Zhou, M.; Memelink, J. Jasmonate-Responsive Transcription Factors Regulating Plant Secondary Metabolism. Biotechnol. Adv. 2016, 34, 441-449. [CrossRef]

58. Li, B.; Zhao, W.; Li, D.; Chao, H.; Zhao, X.; Ta, N.; Li, Y.; Guan, Z.; Guo, L.; Zhang, L.; et al. Genetic Dissection of the Mechanism of Flowering Time Based on an Environmentally Stable and Specific Qtl in Brassica napus. Plant. Sci. 2018, 277, 296-310. [CrossRef]

59. Li, H.; Fan, Y.; Yu, J.; Chai, L.; Zhang, J.; Jiang, J.; Cui, C.; Zheng, B.; Jiang, L.; Lu, K. Genome-Wide Identification of Flowering-Time Genes in Brassica Species and Reveals a Correlation between Selective Pressure and Expression Patterns of Vernalization-Pathway Genes in Brassica napus. Int. J. Mol. Sci. 2018, 19, 3632. [CrossRef]

60. Bernier, G.; Perilleux, C. A Physiological Overview of the Genetics of Flowering Time Control. Plant. Biotechnol. J. 2005, 3, 3-16. [CrossRef]

61. Vega, A.; O’Brien, J.A.; Gutierrez, R.A. Nitrate and Hormonal Signaling Crosstalk for Plant Growth and Development. Curr. Opin. Plant. Biol. 2019, 52, 155-163. [CrossRef] [PubMed]

62. Wen, Z.Y.; Tyerman, S.D.; Dechorgnat, J.; Ovchinnikova, E.; Dhugga, K.S.; Kaiser, B.N. Maize NPF6 Proteins Are Homologs of Arabidopsis Chl1 That Are Selective for Both Nitrate and Chloride. Plant. Cell 2017, 29, 2581-2596. [CrossRef]

63. Wang, W.; Hu, B.; Yuan, D.; Liu, Y.; Che, R.; Hu, Y.; Ou, S.; Liu, Y.; Zhang, Z.; Wang, H.; et al. Expression of the Nitrate Transporter Gene sNRT1.1A/OsNPF6.3 Confers High Yield and Early Maturation in Rice. Plant. Cell 2018, 30, 638-651. [CrossRef] [PubMed]

64. Wang, Y.Y.; Tsay, Y.F. Arabidopsis Nitrate Transporter NRT1.9 Is Important in Phloem Nitrate Transport. Plant. Cell 2011, 23, 1945-1957. [CrossRef] [PubMed]

65. Tang, W.J.; Ye, J.; Yao, X.M.; Zhao, P.Z.; Xuan, W.; Tian, Y.L.; Zhang, Y.Y.; Xu, S.; An, H.Z.; Chen, G.M.; et al. Genome-Wide Associated Study Identifies Nac42-Activated Nitrate Transporter Conferring High Nitrogen Use Efficiency in Rice. Nat. Commun. 2019, 10, 5279. [CrossRef] [PubMed]

66. Pike, S.; Gao, F.; Kim, M.J.; Kim, S.H.; Schachtman, D.P.; Gassmann, W. Members of the NPF3 Transporter Subfamily Encode Pathogen-Inducible Nitrate/Nitrite Transporters in Grapevine and Arabidopsis. Plant. Cell Physiol. 2014, 55, 162-170. [CrossRef] [PubMed]

67. David, L.C.; Berquin, P.; Kanno, Y.; Seo, M.; Daniel-Vedele, F.; Ferrario-Mery, S. N Availability Modulates the Role of NPF3.1, a Gibberellin Transporter, in GA-Mediated Phenotypes in Arabidopsis. Planta 2016, 244, 1315-1328. [CrossRef]

68. Tal, I.; Zhang, Y.; Jorgensen, M.E.; Pisanty, O.; Barbosa, I.C.R.; Zourelidou, M.; Regnault, T.; Crocoll, C.; Olsen, C.E.; Weinstain, R.; et al. The Arabidopsis NPF3 Protein Is a GA Transporter. Nat. Com. 2016, 7. [CrossRef]

69. Tamura, K.; Stecher, G.; Peterson, D.; Filipski, A.; Kumar, S. MEGA6: Molecular Evolutionary Genetics Analysis Version 6.0. Mol. Biol. Evol. 2013, 30, 2725-2729. [CrossRef]

70. Holub, E.B. The Arms Race Is Ancient History in Arabidopsis, the Wildflower. Nat. Rev. Genet. 2001, 2, 516-527. [CrossRef]

71. Zhang, Z.; Li, J.; Zhao, X.Q.; Wang, J.; Wong, G.K.; Yu, J. Kaks_Calculator: Calculating Ka and Ks through Model Selection and Model Averaging. Genom. Proteom. Bioinform. 2006, 4, 259-263. [CrossRef]

72. Chen, C.; Chen, H.; Zhang, Y.; Thomas, H.R.; Frank, M.H.; He, Y.; Xia, R. Tbtools: An Integrative Toolkit Developed for Interactive Analyses of Big Biological Data. Mol. Plant. 2020, 13, 1194-1202. [CrossRef]

73. Cui, J.Q.; Hua, Y.P.; Zhou, T.; Liu, Y.; Huang, J.Y.; Yue, C.P. Global Landscapes of the Na(+)/H(+) Antiporter (Nhx) Family Members Uncover Their Potential Roles in Regulating the Rapeseed Resistance to Salt Stress. Int. J. Mol. Sci. 2020, $21,3429$.

74. Lu, K.; Li, T.; He, J.; Chang, W.; Zhang, R.; Liu, M.; Yu, M.; Fan, Y.; Ma, J.; Sun, W.; et al. Qprimerdb: A Thermodynamics-Based Gene-Specific qPCR Primer Database for 147 Organisms. Nucleic Acids Res. 2018, 46, 1229-1236. [CrossRef] 